\title{
Default Priors and Predictive Performance in Bayesian Model Averaging, with Application to Growth Determinants*
}

\author{
Theo S. Eicher \\ Department of Economics \\ University of Washington \\ Chris Papageorgiou \\ Research Department \\ International Monetary Fund \\ Adrian E. Raftery \\ Departments of Statistics and Sociology \\ University of Washington
}

April 3, 2009

\begin{abstract}
Bayesian model averaging (BMA) has become widely accepted as a way of accounting for model uncertainty, notably in regression models for identifying the determinants of economic growth. To implement BMA the user must specify a prior distribution in two parts: a prior for the regression parameters and a prior over the model space. Here we address the issue of which default prior to use for BMA in linear regression. We compare 12 candidate parameter priors: the Unit Information Prior (UIP) corresponding to the BIC or Schwarz approximation to the integrated likelihood, a proper data-dependent prior, and 10 priors considered by Fernandez et al. (2001b). We also compare the uniform model prior to others that favor smaller models. We compare them on the basis of crossvalidated predictive performance on a well-known growth dataset and on two simulated examples from the literature. We found that the UIP with uniform model prior generally outperformed the other priors considered. It also identified the largest set of growth determinants.
\end{abstract}

JEL Classification: O51, O52, O53.

Keywords: Growth Determinants; Model Uncertainty; Bayesian Model Averaging (BMA); Parameter and Model Prior Elicitation; Predictive Performance.

\footnotetext{
${ }^{*}$ We thank three anonymous referees, the handling editor (Steven Durlauf), Veronica Berrocal, Gernot Doppelhofer, Edward George, Tilmann Gneiting, Jennifer Hoeting, Andros Kourtellos, Andreas Leukert, Eduardo Ley, Chih Ming Tan, Arnold Zellner, and seminar participants at the Department of Statistics, University of Washington and the 2009 Econometric Society meetings in San Francisco for valuable comments and discussions. We also thank Amanda Cox for her tireless support, advice, and programming, Drew Creal for excellent software programming, Tilmann Gneiting for kindly sharing his CPRS code for BMA applications, Eduardo Ley for sharing data, and Fred Nick at the University of Washington Center for Social Science Computation and Research for providing computing support. Eicher gratefully acknowledges financial support from the University of Washington Center for Statistics and the Social Sciences through a seed grant. Raftery's research was supported by NSF grants ATM 0724721 and IIS-0534094, by NIH grant HD054511 and by the Joint Ensemble Forecasting System (JEFS) under subcontract No. S06-47225 from the University Corporation for Atmospheric Research (UCAR). The views expressed in this study are the sole responsibility of the authors and should not be attributed to the International Monetary Fund, its Executive Board, or its management.
} 


\section{Introduction}

Bayesian model averaging (BMA) is now widely accepted as a principled way of accounting for model uncertainty. ${ }^{1}$ Model uncertainty has played a particularly big role in economic growth research since the early 1990s when a surge of new growth theories gave rise to a large literature that sought to evaluate the new growth determinants (see Durlauf, Johnson and Temple, 2005 for a survey). Linear regression models dominate in growth research, and here we consider BMA for this class of models. The implementation of BMA involves solving the common challenge in Bayesian statistics of specifying the prior. For BMA, the prior has two parts: a prior for the parameters of each model, and the prior probability of each model. ${ }^{2}$ The implementation of BMA is, however, subject to the challenge that it requires prior distributions over all parameters in all models, and the prior probability of each model must also be specified.

If substantial prior information is available and can readily be expressed as a probability distribution, this should be used. Often, however, the prior information is small relative to the information in the data, and then it makes sense to use a default prior. Here we address the issue of which default prior to use.

We compare 12 candidate default parameter priors and two model priors that have been advocated in the literature. We do this on the basis of cross-validated predictive performance using a well-known growth dataset and two simulated examples from the literature. Predictive performance is a natural and neutral basis for such comparisons. We evaluate the predictive mean using the Mean Squared Error, and the entire predictive distribution, using two different scoring rules.

We found substantial support for one of the priors evaluated: the Unit Information Prior (UIP) that corresponds to the BIC (or Schwarz) approximation for the integrated (or marginal) likelihood, combined with a uniform prior over the model space. This also turned out to favor the largest number of growth determinants.

We are not the first to compare priors for BMA in growth regressions. FLS (2001a) applied a "benchmark prior" (FLS 2001b) to the growth context, but did not include the UIP, or alternative model priors. Sala-i-Martin Doppelhofer and Miller (2004, hereafter SDM) compared model prior

\footnotetext{
${ }^{1}$ The economics literature has long recognized model uncertainty as a central problem in regression analyses in general and in growth applications in particular. The initial approach to model selection was to use stepwise regression (Efroymson, 1960). Leamer (1978) suggested extreme bounds analysis to account not only for withinmodel uncertainty, but also for between-model uncertainty, which is associated with model selection (see, Levine and Renelt, 1992, for an application to growth). The BMA methodology was developed by Leamer (1978), Raftery (1988), Madigan and Raftery (1991, 1994) who coined the name, Raftery (1993), George and McCulloch (1993) and others; for a survey of its early development see Hoeting, Madigan, Raftery and Volinsky (1999).

${ }^{2}$ See e.g., Brock and Durlauf (2001), Fernández, Ley and Steel (2001a), Sala-i-Martin, Doppelhofer and Miller (2004), and Ley and Steel (2007a,b)
} 
distributions but did not compare different parameter priors. Ley and Steel (2007b, hereafter LS), following Brown, Vannucci and Fearn (1998; 2002), introduced a hierarchical prior on the model size and integrated out the prior model size in the model averaging. They used two parameter priors that we include in our set of 12 priors below, in combination with fixed and random model priors. However, LS did not include the UIP. ${ }^{3}$

The paper is organized as follows. Section 2 reviews BMA with a focus on prior specification. Section 3 describes how we use predictive performance to compare prior settings and gives results for the growth data. Section 4 gives the results of a simulation experiment, and Section 5 concludes.

\section{Bayesian Model Averaging}

\subsection{Basic Ideas}

We now briefly summarize the main ideas of BMA for linear regression. ${ }^{4}$ Given a dependent variable, $Y$, a number of observations, $n$, and a set of candidate regressors, $X_{1}, \ldots, X_{p}$, the variable selection problem is to find the most effective subset of regressors. We denote by $M_{1}, \ldots, M_{k}$ the models considered, where each one represents a subset of the candidate regressors. When all possible subsets are considered, $K=2^{p}$. Model $M_{k}$ has the form $Y=\alpha+\sum_{j=1}^{p_{k}} \beta_{j}^{(k)} X_{j}^{(k)}+\varepsilon$, where $X_{1}^{(k)}, \ldots, X_{p_{k}}^{(k)}$ is a subset of $X_{1}, \ldots, X_{p}, \beta^{(k)}=\left(\beta_{1}^{(k)}, \ldots, \beta_{p_{k}}^{(k)}\right)$ is a vector of regression coefficients to be estimated, and $\varepsilon \sim N\left(0, \sigma^{2}\right)$ is the error term. We denote by $\theta_{k}=\left(\alpha, \beta^{(k)}, \sigma\right)$ the vector of parameters in $M_{k}$.

The likelihood function of model $M_{k}, \operatorname{pr}\left(D \mid \theta_{k}, M_{k}\right)$, summarizes all the information about $\theta_{k}$ that is provided by the data, $D$. The integrated likelihood (also known as the marginal likelihood) is the probability density of the data, conditional on the model $M_{k}$, which equals the likelihood times the prior density, $\operatorname{pr}\left(\theta_{k} \mid M_{k}\right)$, integrated over the parameter space, so that

$$
\operatorname{pr}\left(D \mid M_{k}\right)=\int \operatorname{pr}\left(D \mid \theta_{k}, M_{k}\right) \operatorname{pr}\left(\theta_{k} \mid M_{k}\right) d \theta_{k}
$$

Equation (1) follows from the law of total probability.

The integrated likelihood is the crucial ingredient in deriving the model weight for model averaging. We denote by $\operatorname{pr}\left(M_{k}\right)$ the prior probability that $M_{k}$ is the correct model, given that one of the models considered is. Then, by Bayes's theorem, the posterior model probability of $M_{k}$,

\footnotetext{
${ }^{3}$ In addition, Durlauf, Kourtellos and Tan (2006; 2008), and Brock, Durlauf and West (2003) evaluated different sets of parameter and model priors; their approaches are discussed below.

${ }^{4}$ Comprehensive surveys of BMA include Raftery, Madigan and Hoeting (1997), Hoeting et al. (1999), Clyde and George (2004), and Doppelhofer (2008)
} 
$\operatorname{pr}\left(M_{k} \mid D\right)$, is equal to the model's share of the total posterior mass,

$$
\operatorname{pr}\left(M_{k} \mid D\right)=\frac{\operatorname{pr}\left(D \mid M_{k}\right) \operatorname{pr}\left(M_{k}\right)}{\sum_{\ell=1}^{K} \operatorname{pr}\left(D \mid M_{\ell}\right) \operatorname{pr}\left(M_{\ell}\right)} .
$$

BMA obtains the posterior inclusion probability of a candidate regressor, $\operatorname{pr}\left(\beta_{j} \neq 0 \mid D\right)$, by summing the posterior model probabilities across those models that include the regressor. Posterior inclusion probabilities provide a probability statement regarding the importance of a regressor that directly addresses what is often the researcher's prime concern: "what is the probability that the regressor has an effect on the dependent variable?" 5

BMA involves averaging over all the models considered. This can be a very large number; for example, the growth dataset we consider below features 41 candidate regressors (and so $K=2^{41}$, or about two trillion models). Such a vast model space involves a major computational challenge as direct evaluation is typically not feasible. In this paper we use the leaps-and-bounds method developed by Raftery (1995) for BMA, based on the all-subsets regression algorithm of Furnival and Wilson (1974). This is implemented in the BMA R package, available at http://cran.r-project.org/ (Raftery et al. 2005, 2009).

Other approaches to dealing with the large model space are the coinflip importance sampling algorithm used by SDM, and the Markov Chain Monte Carlo (MCMC) sampler used by FLS. We have experimented with all three algorithms using the FLS data and found that the results from the branch-and-bound and MCMC methods were very similar, while the coinflip method took substantially more computational time, and produced less precise results. In particular, the coinflip algorithm failed to explore large parts of the model space, notably excluding the models with the highest posterior probabilities.

\section{$2.2 \quad$ Prior Distributions of Parameters}

The implementation of BMA in linear regression is subject to the challenge that prior distributions must be specified over all parameters in all models. Prior probabilities of all models must also be specified. If the researcher has information about the parameters, ideally this should be reflected in the priors, and informative priors should be used, as was done, for example, by Jackman and Western (1994).

\footnotetext{
${ }^{5}$ The posterior inclusion probability will provide an answer to this question only if the regression parameters can be interpreted causally. This will not be the case if, for example, there are common causes of growth and the regressor not included in the model, or if growth affects the regressor rather than the other way round. This issue is the general one of endogeneity and causal interpretation of regression parameters, and is not specific to BMA. We do not consider it further in this paper.
} 
However, often the amount of prior information is small and the effort needed to specify it in terms of a probability distribution is large. Thus there have been many efforts to specify default priors that could reasonably be used for all such analyses. These are sometimes called "noninformative" or "reference" priors, but there is debate about the extent to which a prior can be totally noninformative, and so we use the term "default prior" here. Priors on parameters may affect results since they may influence the integrated likelihood (1), which is a key component of the posterior model weights (2). The integrated likelihood of a model is approximately proportional to the prior density of the model parameter evaluated at the posterior mode (Kass and Raftery, 1995). Thus the prior density should be spread out enough so that it is reasonably flat over the region of the parameter space where the likelihood is substantial. However, the prior density should also be no more spread out than necessary, since increasing the spread of the prior tends to decrease the prior ordinate at the posterior mode, which decreases the integrated likelihood and may unnecessarily penalize larger models (Raftery, 1996). The priors we discuss below make this trade-off in different ways.

We focus on a set of 12 candidate default priors that have been advocated in the literature (Table 1): a prior which contains about the same amount of information as a typical single observation (Kass and Wasserman 1995; Raftery, 1995); the data-dependent prior of Raftery, Madigan and Hoeting (1997), which was designed to be relatively flat over the region of the parameter space supported by the data but no more spread out than necessary; and third, the ten automatic priors used by FLS (2001b), which do not rely on input from the researcher or information in the data, but only on the sample size and the number of regressors.

The first prior that we consider is defined implicitly, by the form of the approximate integrated likelihood that is used, namely,

$$
\log \operatorname{pr}\left(D \mid M_{k}\right) \approx c-\frac{1}{2} B I C_{k}
$$

where

$$
B I C_{k}=n \log \left(1-R_{k}^{2}\right)+p_{k} \log (n) .
$$

In (4), $R_{k}^{2}$ and $p_{k}$ are the coefficient of determination and the number of regressors, respectively, for model $M_{k}$, and $c$ is a constant that does not vary across models and so cancels in the model averaging. $B I C_{k}$ is the Bayesian Information Criterion for $M_{k}$, which is equivalent to the approx- 
imation derived by Schwarz (1978) for the regression model, as shown by Raftery (1995). ${ }^{6}$ The approximate integrated likelihood in (3) was the basis of the model averaging method of Raftery (1995) for linear regression, and was also used by SDM.

Raftery (1995, Section 4) showed that (3) gives an approximation to the integrated likelihood with an error that is $O\left(n^{-1 / 2}\right)$ when the prior for the regression parameters is multivariate normal centered at the maximum likelihood estimate with variance matrix equal to the $n$ times the inverse of the observed Fisher information matrix. ${ }^{7}$ This prior is much more spread out than the likelihood, and typically is relatively flat where the likelihood is substantial (Raftery, 1999). It contains the same amount of information as would be contained on average in a single observation and so, following Kass and Wasserman (1995), we call it the Unit Information Prior (UIP). Because of its simplicity and intuitive appeal, we use the UIP as a baseline, and we compare other proposed default priors to it. ${ }^{8}$

Next, we consider ten automatic priors considered by FLS (2001b) of the following form:

$$
\begin{aligned}
p\left(\alpha \mid M_{k}\right) & \propto 1, \\
p\left(\sigma \mid M_{k}\right) & \propto 1 / \sigma \\
\beta^{(k)} \mid \sigma, M_{k} & \sim N\left(0, \sigma^{2}\left(g_{k} Z^{(k) \prime} Z^{(k)}\right)^{-1}\right),
\end{aligned}
$$

where $Z^{(k)}$ is the $n \times p_{k}$ matrix consisting of the $p_{k}$ regressors included in $M_{k}$, each one centered by subtracting its mean. The prior (7) for $\beta^{(k)}$ is based on Zellner's (1986) $g$-prior, but the overall prior (5)-(7) was proposed by FLS (2001b), who showed that it leads to analytical integrated likelihoods. The value of $g$ scales the reciprocal of the variance of the parameter prior. Values of $g$ that are closer to zero imply priors that are less informative, and $g=1$ implies that prior information and data information are weighted equally in the posterior distribution. Different automatic priors result from different choices of $g_{k}$, as listed in Table 1 .

\footnotetext{
${ }^{6}$ Klein and Brown (1984) discuss an alternative derivation of BIC model weights by minimizing the Shannon information in the prior distribution.

${ }^{7}$ It follows further from the results of Kass and Wasserman (1995) that for any pairwise model comparison, the ratio of posterior model probabilities resulting from the use of (3) closely approximates the ratio of posterior model probabilities with a prior that is the same except that its mean is equal to zero instead of the MLE, again with error of order $O\left(n^{-1 / 2}\right)$.

${ }^{8}$ It could be argued that this prior depends on the data and so is not a valid prior for Bayesian analysis. However, we use it here as an approximation to the prior information of an analyst who knows something, but not a great deal about the problem at hand. For estimating a population mean, its use implies roughly that the analyst knows at least that the mean is within the range of the data, and it seems likely that anyone analyzing data about the problem would know at least that much (Raftery 1999). Wasserman (2000) showed that data-dependent priors can actually improve predictive performance. FLS (2001b) point out a common criticism of data-dependent priors, namely that the posterior distribution can no longer be interpreted as a conditional distribution given the observables.
} 
The choice $g=1 / n$ (Prior 12 in Table 1 ) has the same variance as the UIP, but its mean is at zero instead of the MLE. Alternatives are Prior $4, g=\sqrt{1 / n}$, which attributes a smaller asymptotic penalty than BIC, and Prior $2, g_{k}=p_{k} / n$, where prior information increases with the number of regressors in the model. Other priors suggested by FLS (2001b) correspond to previous proposals: Priors 6 and 7 in Table 1 are versions of the Hannan and Quinn criterion (Hannan and Quinn, 1979), and Prior 9, $g_{k}=1 / p^{2}$, corresponds to the Risk Inflation Criterion (RIC) of Foster and George (1994), designed to take account of the number of candidate regressors. Prior 10 is the preferred prior of FLS (2001b), developed on the basis of their experiments with their priors. It is composed of either the RIC-based prior (Prior 9) or Prior 12, depending on the number of observations and regressors in the particular dataset. For the datasets considered in this paper, Prior 10 is identical to Prior 9.

An alternative class of data-dependent priors can be viewed as approximating the subjective prior of an experienced researcher. Clearly, if such knowledge can be readily elicited in the form of a probability distribution, it should be introduced into the analysis, Raftery, Madigan and Hoeting (1997) specified conjugate data-dependent priors that are as concentrated as possible, subject to being reasonably flat over the region of parameter space where the likelihood is not negligible. Their prior (Table 1, Prior 11) is specified by four hyperparameters that are explained in Table 1. Another such data-dependent prior is based on Laud and Ibrahim (1996) (Table 1, Prior 8) who specified $g=\delta \gamma^{1 / p_{j}} /\left(1-\delta \gamma^{1 / p_{j}}\right)$. Given FLS's suggestions for $\gamma$ and $\delta$, they mention that model comparisons based on the resulting log integrated likelihood can roughly be compared to those based on the Akaike Information Criterion (AIC) (Akaike, 1974).

\subsection{Model Priors}

The most common model prior in the literature is the uniform distribution that assigns equal prior probability all models, so that $\operatorname{pr}\left(M_{k}\right)=1 / K$ for each $k$. This was suggested first by Raftery (1988) and, for linear regression models, by George and McCulloch (1993). Hoeting et al. (1999) cite the extensive evidence that supports the good performance of the UIP, since the integrated likelihood on the model space is often concentrated enough for the results to be insensitive to moderate deviations from the uniform prior.

We also consider the more general model prior proposed by Mitchell and Beauchamp (1988), namely

$$
\operatorname{pr}\left(M_{k}\right)=\prod_{j=1}^{p} \pi_{j}^{\delta_{k j}}\left(1-\pi_{j}\right)^{1-\delta_{k j}}
$$


where $\delta_{k j}=1$ if $X_{j}$ is included in $M_{k}$ and 0 otherwise. In (8), $\pi_{j}$ is the prior probability that $X_{j}$ is included in the model, and it is usually assumed that $\pi_{j}=\pi$ for $j=1, \ldots, p$. When $\pi=0.5$, (8) reduces to the uniform prior. The general prior in (8) has been widely used, for example by George and McCulloch (1993), Madigan and Raftery (1994) and SDM. SDM assumed $\pi=7 / p$ in growth applications, yielding a prior expected model size of $\pi p=7$. Following Brown, Vannucci, and Fearn (1998; 2002), Ley and Steel (2007b, hereafter LS) suggested that $\pi$ itself be a random variable drawn from a $\operatorname{Beta}\left(1, \frac{1-\pi}{\pi}\right)$ distribution. They evaluated parameter Priors 9 and 12 with fixed and random $\pi$. We adopt (8) and examine growth determinants as well as their predictive performance for a range of fixed model priors. We also compare our results with those in LS with fixed and random $\pi .^{9}$

There is a tradeoff between the prior inverse variance parameter $g$ and the prior inclusion probabilities, $\pi_{j}$ in (8), pointed out by Taplin and Raftery (1994, Section 5.2) in a slightly different context, and also revealed by computations in Ley and Steel (2007b). We now give a theoretical explanation for this, taking $\pi_{j}=\pi$ for $j=1, \ldots, p$ for easier exposition.

Comparing the posterior probabilities for a given model in (2) for different priors, Kass and Raftery (1995) showed that an increase in the prior standard deviation by a factor $c$, is approximately equivalent to a reduction in the prior odds for an increase in the model size by an additional variable, by the same factor of $c$.

Using the approximation of Kass and Raftery (1995, equation 14), it can be shown that for two priors, $A$ and $B$, with associated prior scale factors, $g_{A}, g_{B}$, and prior inclusion probabilities, $\pi_{A}$, $\pi_{B}$, the posterior odds for one regression model against an alternative regression model with one additional regressor are approximately equal when the priors satisfy

$$
\frac{g_{A}}{g_{B}}=\left[\frac{\pi_{B}}{\pi_{A}} \frac{\left(1-\pi_{A}\right)}{\left(1-\pi_{B}\right)}\right]^{2}
$$

This shows the nature of the tradeoff between the prior scale factor and the prior inclusion probability: a change in $\pi$ has approximately the same effect as the change in $g$ given by equation (9).

\footnotetext{
${ }^{9}$ Like most workers in this area, we use independent the independent model priors specified by (8). However, non-independent default priors have been proposed as well. George (1999; 2001) and Durlauf, Kourtellos and Tang (2008) introduced dependent model priors that account for the correlation structure of the regressors. Brock, Durlauf and West (2003) proposed tree-structured priors that are based on substantive knowledge of context.
} 


\section{Determining Growth Determinants}

Since economic growth is the fundamental driver of living standards, it is of great interest to economists and policymakers alike to identify which of the numerous theories proposed receive support from the data and which determinants have a significant effect on growth. Attempts to identify robust growth determinants date back to Levine and Renelt (1992) who used extreme bounds analysis. Formal BMA analysis was conducted by Brock and Durlauf (2001), FLS (2001a) and SDM (2004). The dataset used across studies always contains a core of at least 41 candidate regressors, motivated by Sala-i-Martin (1997) and FLS (2001a). We base our growth analysis on the same dataset that FLS kindly shared with us.

\subsection{Effects of Parameter Priors on Growth Determinants}

For datasets with small numbers of observations such as our growth dataset with 72 observations, priors can play an important role. As can be seen in Figure 1, the precisions of the parameter priors vary widely; for example the information contained in Prior 7 is three orders of magnitude greater than that in the FLS-preferred prior. It thus seems possible that the BMA results would vary considerably between priors.

Table 2 reports the BMA posterior inclusion probabilities for all 12 prior distributions applied to the growth dataset. Posterior inclusion probabilities and the number of regressors that exhibit evidence of an effect on growth vary substantially across priors. The number of regressors whose inclusion probability exceeds $50 \%$ ranges from a low of 7 regressors (Priors 5, 7, and 11) to a high of 22 regressors (Prior 1). Recall that, apart from the UIP, the prior distributions are all centered at zero and that Priors 5, and 7 have small prior variance that emphasizes the zero expected mean, while the variance of Prior 11 has the largest variance in the sample, which to emphasize uncertainty (see Figure 1). Priors 5, 7, and 11 contain strong information against a large effect, and the information contained in the data is too weak to overwhelm that prior. As the priors over the parameter space become spread out enough to include those regions where the likelihood is large, the number of regressors that exhibit an effect increases. Figure 1 shows that both more diffuse and more precise priors (Priors 11, 7 and 5) lead to a decline in the integrated likelihood, thus reducing the number of regressors showing an effect.

Figure 2 shows scatterplots of posterior inclusion probabilities generated by the various priors against Prior 1 . Since Prior 1 was the most optimistic, with 22 candidate regressors showing an effect in Table 2, it is no surprise that most of the points in the scatterplots lie above the 45 degree 
line, indicating higher posterior inclusion probabilities under Prior 1 than under other priors. The scatterplots also show how the differences between Prior 1 and alternative priors increase as the implied $g$-prior diverges. Priors 1, 6, and 12 yielded similar results, but most other priors showed differing effects implied by the priors.

\subsection{Combined Effects of Parameter and Model Priors on Growth Determinants}

SDM advocated using a Mitchell-Beauchamp prior (8) with $\pi=7 / p$, equivalent to a prior expected model size of 7 regressors. We combined this model prior with the 12 parameter priors considered, and the results are shown in Table 3. As expected, this leads to smaller models than the uniform model prior, ranging from 3 to 10 effective regressors with posterior inclusion probabilities above $50 \% .{ }^{10}$ Again the priors with intermediate variance have a slightly larger number of regressors (Priors 3, 4, and 12), and as before the number of regressors that exhibit an effect declines as the prior variance become large (Priors 6 and 9). The Mitchell-Beauchamp model prior has the least impact on Prior 11; for this prior, the Rule of Law variable loses significance but otherwise the results are identical to Table 2.

The image plots in Figure 3, produced by the BMA R package, highlight how different the models are over which the various priors average. The figure shows models used in the averaging process on the horizontal axis. Each model's posterior probability is indicated by its horizontal width. Red and blue indicate the inclusion of a regressor with a positive and negative posterior mean, respectively. Comparing Figures 3a and 3c, we see that the model prior with prior expected model size 7 favors growth models with fewer variables. In addition, the image plots highlight that, while the procedure averages over the same number of models, many more models receive negligible weight if the model size is presumed to be small. On the other hand, we note the similarity between Figures $3 \mathrm{~b}$ and $3 \mathrm{c}$, that feature two very different model and parameter priors. This similarity was first observed for these specific priors by Masanjala and Papageorgiou (2005). LS describe the similarity between the FLS uniform prior and Prior 1 with prior model size 7 as arising "mostly by accident" and discuss specific parameter constellations that generate similar posterior probabilities. We showed in Section 2.3 that in fact this similarity has a theoretical explanation.

For the FLS dataset with $n=72$ and $p=41$, the FLS benchmark parameter prior implies $g_{A}=1 / p^{2}$, combined with the uniform model prior, $\pi_{A}=\frac{1}{2}$, in the notation of $(9)$. When $g_{B}=1 / n$

\footnotetext{
${ }^{10}$ When the posterior inclusion probability is above $50 \%$, the Bayesian analysis favors inclusion because the coefficient is more likely than not to be in the model, given the data. There is also some theoretical support for emphasizing predictor variables whose posterior inclusion probability is above $50 \%$, namely that the so-called "median probability model" that includes precisely those variables is often the optimal predictive model (Barbieri and Berger 2004).
} 
as in the case of Prior 1, used by SDM, equation (9) holds when the prior inclusion probability is $\pi_{A}=7.03 / p$, so that the prior expected model size is 7.03. It is therefore not surprising that for the SDM suggested prior expected model size of 7, the priors recommended by SDM and FLS yield similar results for the growth dataset, although they are based on very different parameter and model priors. Note that this similarity depends crucially on the number of candidate regressors in the dataset, $p$. Subjective priors that favor small models thus achieve their aim by punishing larger models (Figure 3c) or by increasing the prior variance on each individual parameter (Figure 3b).

In summary, candidate default priors differed considerably in dispersion, and led to the choice of different sets of variables. As few as 3 and as many as 22 regressors were found to be related to growth, depending on the specific prior used.

\subsection{Assessment of Prior Distributions using Predictive Performance}

We now compare the competing default priors on the basis of predictive performance on hold-out samples, a neutral criterion that allows the comparison of different methods on the same footing. We compare the performance of the full predictive distributions produced by the methods, as well as that of point predictions.

We divide the dataset randomly into a training set, $D^{T}$, which is used to estimate the BMA predictive distribution, and a hold-out set, $D^{H}$, which is used to assess the quality of the resulting predictive distributions. We use three different criteria, or scoring rules: the mean squared error (MSE) of prediction, the log predictive score (LPS; Good 1952), and the continuous ranked probability score (CRPS; Matheson and Winkler, 1976). All our scoring rules are negatively oriented, that is, lower is better.

The MSE of prediction is conventionally used to assess the quality of point predictions. The BMA point prediction for an observation in the hold-out dataset, $y_{\text {new }}$, with predictors $x_{\text {new }}$, is

$$
\hat{y}_{\text {new }, \text { BMA }}=\sum_{k=1}^{K} E\left[y_{\text {new }} \mid x_{\text {new }}, D^{T}, M_{k}\right] \operatorname{pr}\left(M_{k} \mid D^{T}\right) .
$$

The MSE of prediction is then

$$
\frac{1}{n_{H}} \sum_{y_{\text {new }} \in D^{H}}\left(y_{\text {new }}-\hat{y}_{\text {new,BMA }}\right)^{2}
$$

where $n_{H}$ is the number of observations in $D^{H}$.

The other two scoring rules measure the quality of the predictive distribution as a whole. The 
BMA predictive distribution is

$$
\operatorname{pr}_{\mathrm{BMA}}\left(y_{\text {new }}\right)=\sum_{k=1}^{K} \operatorname{pr}\left(y_{\text {new }} \mid x_{\text {new }}, D^{T}, M_{k}\right) \operatorname{pr}\left(M_{k} \mid D^{T}\right) .
$$

The LPS is then defined as

$$
L P S=-\sum_{y_{\text {new }} \in D^{H}} \log p r_{\mathrm{BMA}}\left(y_{\text {new }}\right)
$$

Let $F_{\mathrm{BMA}}\left(y_{\text {new }}\right)$ be the cumulative distribution function corresponding to the BMA predictive density $\operatorname{pr}_{\mathrm{BMA}}\left(y_{\text {new }}\right)$. Then the CRPS for the single observation $y_{\text {new }}$ is

$$
\operatorname{CRPS}\left(y_{\mathrm{new}}\right)=\int_{-\infty}^{\infty}\left(F_{\mathrm{BMA}}(y)-1\left\{y_{\text {new }}<y\right\}\right)^{2} d y
$$

where $1\left\{y_{\text {new }}>y\right\}=1$ if $y_{\text {new }}>y$ and 0 otherwise. The CRPS for the hold-out dataset as a whole is then

$$
C R P S=\frac{1}{n_{H}} \sum_{y_{\text {new }} \in D^{H}} C R P S\left(y_{\text {new }}\right) .
$$

The CRPS measures the area between a step function at the observed value and the predictive cumulative distribution function. Unlike the LPS, it is defined when the prediction is deterministic; in that case it reduces to the mean absolute error (Hersbach 2002).

The LPS and the CRPS assess both the sharpness of a predictive distribution and its calibration, namely the consistency between the distributional forecasts and the observations. However, the LPS assigns particularly harsh penalties to poor probabilistic forecasts, and so can be very sensitive to outliers and extreme events (Weigend and Shi, 2000; Gneiting and Raftery, 2007). The CRPS is more robust to outliers (Carney, Cunningham and Byrne, 2006; Gneiting and Raftery, 2007), and hence it is our preferred measure of the performance of the predictive distribution as a whole. We also report the LPS for comparability with previous work, notably that of FLS (2001b) and LS.

We divided the dataset randomly into a training set that contains $80 \%$ of the data and thus leaves $20 \%$ of the data to be predicted, and we repeated the analysis for 400 different random splits, reporting the average over all splits. Table 4a shows the predictive performance of the 12 parameter priors in conjunction with uniform model priors as evaluated by the MSE, LPS and CRPS.

The MSE and the CRPS agree that our baseline Prior 1 decisively outperformed all the other priors. The LPS suggests, however, that Priors 2, 4, 6 and 8 outperform Prior 1. Since this result runs counter to the results from the two other scoring rules, it seems possible that the difference is due to influential observations in the dataset or outliers in a particular subsample. Several of the regressors have extreme outlying values. When such cases are in the test set, they can have a large 
effect on the LPS, while the CRPS is more robust to individual cases. Given the known outlier sensitivity of the LPS, we discount the results it gives for this dataset, and conclude that Prior 1 performs best in this case.

Table 4b compares our results to those of LS (Table 5) who did not consider the UIP, but who did include random model priors for parameter priors 9 and 12, in which a prior distribution was put on the prior inclusion probability $\pi$. To achieve an exact comparison with the LS results, Table $4 \mathrm{~b}$ is based on a $85 / 15$ subsample split and we divide our LPS values by the number of held out observations (following LS's LPS formula). In addition, we report absolute log predicitve scores (LPS) in Table 4b (not values relative to the UIP LPS scores as we do in all of our other tables). Table $4 \mathrm{~b}$ shows that Prior 1 outperformed Priors 9 and 12, whether the model priors are fixed or random.

Recall from Table 7a that Prior 1 had better (lower) LPS values than either Prior 9 or Prior 12 with uniform model priors. LS then show that uniform or random model priors generate similar means for Priors 9 and 12. Hence it is no surprise that UIP also has lower LPS values than Priors 9 or 12 with random model priors.

Overall, the unit information prior (Prior 1) with a uniform model prior performed best of the candidate default priors that we have evaluated in terms of cross-validated predictive performance on the growth dataset. Also, the prior expectation of a model size of about 7 regressors is not supported by the predictive performance results, as shown in Table 5 . Indeed in the growth context predictive performance does not support researchers' priors for small growth models. Table 5 shows that parameter Prior 1 together with uniform model priors dominate all other priors for prior model size smaller than 13 .

\section{Simulated Data}

We now examine the effects of the set of priors using simulated datasets from two models that have been prominent in the BMA literature: Model 1 that is based on Raftery, Madigan and Hoeting (1997) and was used by FLS, and Model 2 that is based on George and McCulloch (1993) was also used by FLS.

For Model 1 we generate an $n \times p(p=15)$ matrix $R=\left(r_{1}, \ldots, r_{15}\right)$ of regressors, where the first ten columns are drawn from independent standard normal distributions, and the next five columns are constructed according to $\left(r_{11}, \ldots, r_{15}\right)=\left(r_{1}, \ldots, r_{5}\right)\left(\begin{array}{lllll}0.3 & 0.5 & 0.7 & 0.9 & 1.1\end{array}\right)^{\prime}(1,1,1,1,1)+E$, where $E$ is an $n \times 5$ matrix of independent standard normal deviates. Model 1 implies small to moderate 
correlations between the first and last five regressors $r_{1}, \ldots, r_{5}$ and $r_{11}, \ldots, r_{15}$. The correlations increase from 0.153 to 0.561 for $r_{1}, \ldots, r_{5}$ and are somewhat larger between the last five regressors, reaching 0.740. Each regressor is centered by subtracting its mean, which results in a matrix $Z=\left(z_{1}, \ldots, z_{15}\right)$. A vector of $n$ observations is then generated according to

$$
\text { Model 1: } 1: y=4 i_{n}+2 z_{1}-z_{5}+1.5 z_{7}+z_{11}+0.5 z_{13}+\sigma \varepsilon
$$

where the $n$ elements of $\varepsilon$ are independent standard normal and $\sigma=2.5$. In Model 1 a third of all the regressors intervene, which we view as fairly typical of some real world situations, and we examine datasets with 50 and 100 observations to stay close to the structure of our growth example.

The structure of Model 2 is closer to the growth dataset in terms of numbers of observations and numbers of regressors. It is generated using $p$ regressors, $r_{i}=r_{i}^{*}+e, i=1, \ldots, p$, where $r_{i}^{*}$ and $e$ are $n$-dimensional vectors of independent standard normal deviates. This induces a pairwise correlation of 0.5 between all regressors. Let $Z$ again denote the $n \times p$ matrix of centered regressors, and generate the $n$ observations according to

$$
\text { Model 2: } y=i_{n}+\sum_{h=1}^{p / 2} z_{(p / 2+h)}+\sigma \varepsilon,
$$

where the $n$ elements of the error are again independent standard normal and $\sigma=2$. In this simulation model, the second half of the regressors intervene, namely $\left(z_{21}, \ldots, z_{40}\right)$.

For Model 1, the differences in the prior variances shown in Figures 4a,b,c are similar to the magnitudes observed for the growth dataset in Figure 1. Again about three orders of magnitude separate the most concentrated and most diffuse priors, although the level of concentration is a bit lower in the simulated datasets. Tables $6 \mathrm{a}, \mathrm{b}$ show, however, that with well-behaved data all priors basically agree upon which regressors have an effect, even in a dataset that contains only 50 observations. For the larger simulated dataset in Model 2, with about three times the number of candidate regressors as in Model 1, we again find diversity in the number of regressors identified as having an effect on the dependent variable. Table $6 \mathrm{c}$ shows that several priors are clearly too concentrated, with Priors 2,5 , and 7 identifying only between 3 and 7 of the 20 relevant regressors that in fact had an effect on the dependent variable. As the prior variance increases enough to cover the more substantive part of the likelihood, the priors are able to pick up more of the relevant regressors, getting closer to the correct number of regressors. Priors 3, 9, and 11 pick up 16 candidate regressors although only Prior 1 shows appropriately high posterior inclusion probabilities. 
In summary, our simulation experiment shows that priors can matter, especially when there are many candidate regressors. The UIP is the only one that was robust across simulations, coming closest to identifying the right regressors in all cases.

Table 7 shows the UIP's generally superior predictive performance. The MSE was consistently better for the UIP than for all other priors. The LPS was too, except for prior 3 in Model 2. The CRPS preferred the UIP to all other priors for Model 2, but for Model 1 it preferred Priors 3, 4, 6 and 8 to Prior 1.

\section{Conclusion}

Model uncertainty is intrinsic in economic analysis and the economic growth literature has been a showcase for model uncertainty over the past decade. Over 140 growth determinants have been motivated by the empirical literature, and the number of competing theories has grown dramatically since the advent of the New Growth Theory. Bayesian Model Averaging (BMA) provides a solid theoretical foundation for addressing model uncertainty as part of the empirical strategy.

However, BMA faces an important challenge. In this paper we showed that for a well-known growth dataset the results of BMA were sensitive to the prior specification. To identify the best prior for our growth dataset, we examined the predictive performance of 12 candidate default parameter priors that have been proposed in the statistics and economics literatures, as well as several model priors that have been advocated. We argue that predictive performance is a natural and neutral criterion for comparing different priors, and suggest the CRPS as a preferred measure. In addition, we examined these priors' success in identifying the right determinants in simulated datasets.

The UIP performed better than the other 11 priors in the growth data, and in simulated data, and as measured by our preferred CRPS scoring rule. The UIP together with the uniform model prior also performed better than the Mitchell-Beauchamp model prior with expected model size 7, which had previously been recommended by Sala-i-Martin, Doppelhofer and Miller (2004). We view the UIP with the uniform model prior as a reasonable default prior and starting place, but our results also highlight that researchers should also assess other possibilities that may be more appropriate for their data and applications.

We have focused here on priors where $\pi$ and $g$ are fixed. A Bayesian alternative is to put prior distributions on $\pi$ and $g$ themselves and integrate them out. Ley and Steel (2007) advocated putting a prior distribution on $\pi$ but their results did not show that this led to improved predictive 
performance, as we have seen. Liang et al. (2008) reviewed a range of parameter priors that put a prior on $g$ and integrate it out (they called them mixtures of $g$-priors). They assessed predictive performance in one example using only the highest probability model under each prior rather than BMA, and reported only the MSE of prediction, and not any measure of the performance of the full predictive distribution. They concluded that the differences in MSE were not enough to suggest that the mixtures of $g$-priors performed better than the fixed $g$ methods. It would be interesting to see a more complete assessment of these methods in terms of predictive performance.

In terms of economic impact, the UIP with uniform model prior identified more growth determinants than Fernández, Ley and Steel (2001b) who used the same dataset. The additional regressors include Primary and Secondary Education, Size of Labor Force, Ethnolinguistic Fragmentation, Minging, Latin America, Colonies (British, French, Spanish), Civil Liberties, Non Equipment Investment, Black Market Premium, Outward Orientation and Fraction Speaking English and Hindu.

\section{References}

Acemoglu, D., S. Johnson and J. Robinson. (2001). "The Colonial Origins of Comparative Development: An Empirical Investigation," American Economic Review 91, 1369-1401.

Akaike, H. (1974). "A New Look at the Statistical Model Identification". IEEE Transactions on Automatic Control 19, 716-723.

Barbieri, M.M. and Berger, J.O. (2004). Optimal predictive model selection. Annals of Statistics $32,870-897$.

Brier, G. (1950). "Verification of Forecasts Expressed in Terms of Probability," Monthly Weather Review 78, 1-3.

Brock, W. and S.N. Durlauf. (2001). "Growth Empirics and Reality," The World Bank Economic Review 15, 229-272.

Brock, W., S.N. Durlauf and K. West. (2003). "Policy Evaluation in Uncertain Economic Environments," Brookings Papers on Economic Activity 1, 235-322.

Brown, P.J., M. Vannucci, and T. Fearn. (1998). "Multivariate Bayesian Variable Selection and Prediction," Journal of the Royal Statistical Society. Series B 60, 627-641.

Brown, P.J., M. Vannucci, and T. Fearn. (2002). "Bayes Model Averaging with Selection of Regressors," Journal of the Royal Statistical Society, Series B 64, 519-536.

Carney, M., P. Cunningham and S. Byrne. (forthcoming). "The Benefits of Using a Complete Probability Distribution when Decision Making: An Example in Anticoagulant Drug Therapy," Medical Decision Making.

Clyde, M. and E.I. George. (2004). "Model Uncertainty," Statistical Science 19, 81-94. 
Clyde, M., H. DeSimone and G. Parmigiani. (1996). "Prediction Via Orthogonalized Model Mixing," Journal of the American Statistical Association 91, 1197-1208.

Doppelhofer, G. (2008). "Model Averaging" in The New Palgrave Dictionary in Economics, 2nd edition. L. Blume and S. Durlauf (eds.).

Durlauf, S.N., P. Johnson and J. Temple. (2005). "Growth Econometrics," in Handbook of Economic Growth, P. Aghion and N. Durlauf, eds., North Holland, Amsterdam.

Durlauf, S.N., P. Johnson and J. Temple. (2009, forthcoming). "The Methods of Growth Econometrics," in Palgrave Handbook of Econometrics, Vol. 2, ed. by T.C. Mills and K. Patterson (London: Palgrave MacMillan).

Durlauf, S.N., A. Kourtellos and C.-M. Tan. (2006). "Is God in the Details? A Reexamination of the Role of Religion in Economic Growth," working paper, University of Wisconsin.

Durlauf, S.N., A. Kourtellos and C.-M. Tan. (2008). "Are Any Growth Theories Robust?" Economic Journal 118, 329-346.

Efroymson, M.A. (1960). "Multiple Regression Analysis," in Mathematical Methods for Digital Computers, edited by A. Ralston and H.S. Wilf. Wiley, New York.

Fernández C., E. Ley and M.F.J. Steel. (2001a). "Model Uncertainty in Cross-Country Growth Regressions," Journal of Applied Econometrics 16, 563-576.

Fernández C., E. Ley and M.F.J. Steel. (2001b). "Benchmark Priors for Bayesian Model Averaging," Journal of Econometrics 100, 381-427.

Foster, D.P. and E.I. George. (1994). "The Risk Inflation Criterion for Multiple Regression," The Annals of Statistics 22, 1947-1975.

Furnival G.M. and R.W. Wilson. (1974). "Regressions by Leaps and Bounds," Technometrics 16, 499-511.

George, E.I. (1999). "Sampling Considerations for Model Averaging and Model Search," Invited discussion of "Bayesian Model Averaging and Model Search Strategies by M.A. Clyde". Bayesian Statistics, 6, (J.M. Bernardo, J.O. Berger, A.P. Dawid and A.F.M. Smith, eds.), Oxford University Press.

George, E.I. (2001). "Dilution Priors For Model Uncertainty," University of Texas MSRI Workshop on Nonlinear Estimation and Classification, Berkeley, California.

George, E.I. and R.E. McCulloch. (1993). "Variable Selection via Gibbs Sampling," Journal of the American Statistical Association 88, 881-889.

Gneiting, T., Balabdaoui, F. and Raftery, A.E. (2007). "Probabilistic Forecasts, Calibration and Sharpness," Journal of the Royal Statistical Society, Series B 69, 243-268.

Gneiting, T. and A.E. Raftery. (2007). "Strictly Proper Scoring Rules, Prediction and Estimation," Journal of the American Statistical Association 102, 359-378. 
Good, I.J. (1952). "Rational Decisions," Journal of the Royal Statistical Society, Series B 14, $107-114$.

Hannan, E.J. and B.G. Quinn. (1979). "The Determination of the Order of an Autoregression," Journal of the Royal Statistical Society, Series B 41, 190-195.

Hersbach, H. (2002). "Decomposition of the Continuous Ranked Probability Score for Ensembles Prediction Systems," Weather and Forecasting 15, 559-570.

Hoeting, J.A., D. Madigan, A.E. Raftery and C.T. Volinsky. (1999). "Bayesian Model Averaging: A Tutorial," Statistical Science 14, 382-417.

Jackman S. and B. Western. (1994). "Bayesian Inference for Comparative Research," American Political Science Review 88, 412-23.

Jeffreys, H. (1961). The Theory of Probability. Oxford University Press. Journal of the American Statistical Association 91, 1197-1208.

Kass, R.E. and A.E. Raftery. (1995). "Bayes Factors," Journal of the American Statistical Association 90, 773-795.

Kass, R.E. and L. Wasserman. (1995). "A Reference Bayesian Test for Nested Hypotheses and its Relationship to the Schwarz Criterion," Journal of the American Statistical Association 90, 928-934.

Klein RW and S.J. Brown. (1984). "Model Selection When There is Miminal Prior Information," Econometrica 52, 1291-1321.

Laud, P.W. and J.G. Ibrahim. (1996). "Predictive Specification of Prior Model Probabilities in Variable Selection," Biometrika 83, 267-274.

Leamer, E.E. (1978). Specification Searches: Ad Hoc Inference with Nonexperimental Data, Wiley, New York.

Levine, R. and D. Renelt. (1992). "A Sensitivity Analysis of Cross-Country Growth Regressions," American Economic Review 82, 942-963.

Ley, E. and M.F.J. Steel. (2007a). "Jointness in Bayesian Variable Selection with Applications to Growth Regression," Journal of Macroeconomics 29, 476-493.

Ley, E. and M.F.J. Steel. (2007b, forthcoming). "On the Effect of Prior Assumptions in BMA with Applications to Growth Regression," Journal of Applied Econometrics.

Liang, F., Paulo, R, German, G., Clyde, M.A. and Berger, J.O. (2008). "Mixtures of $g$ Priors for Bayesian Variable Selection." Journal of the American Statistical Association 103, 401-414.

Madigan, D. and A.E. Raftery. (1991). "Model Selection and Accounting for Model Uncertainty in Graphical Models using Occam's Window." Technical Report no. 213, Department of Statistics, University of Washington, Seattle, Wash. 
Madigan, D. and A.E. Raftery. (1994). "Model Selection and Accounting for Model Uncertainty in Graphical Models using Occam's Window," Journal of the American Statistical Association 89, 1535-1546.

Mankiw, N.G., D. Romer and D.N. Weil. (1992). "A Contribution to the Empirics of Economic Growth," Quarterly Journal of Economics 107, 407-437.

Masanjala, W.H. and C. Papageorgiou. (2005). "Initial Conditions, European Colonialism and Africa's Growth," working paper, Louisiana State University.

Matheson, J. and R. Winkler. (1976). "Scoring Rules for Continuous Probability Distributions," Management Science 22, 1087-1095.

Mitchell, T.J. and J.J. Beauchamp. (1988). "Bayesian Variable Selection in Linear Regression (with discussion)," Journal of the American Statistical Association 83, 1023-1036.

Raftery, A.E. (1988). "Approximate Bayes Factors for Generalized Linear Models," Technical Report no. 121, Department of Statistics, University of Washington.

Raftery, A.E. (1993). "Bayesian Model Selection in Structural Equation Models," in Testing Structural Equation Models (K.A. Bollen and J.S. Long, eds.), Beverly Hills: Sage.

Raftery, A.E. (1995). "Bayesian Model Selection for Social Research," Sociological Methodology $25,111-163$.

Raftery, A.E. (1996). "Approximate Bayes Factors and Accounting for Model Uncertainty in Generalized Linear Models." Biometrika 83, 251-266.

Raftery, A.E. (1999). "Bayes Factors and BIC: Comment on Weakliem," Sociological Methods and Research 27, 411-427.

Raftery, A.E., D. Madigan and J.A. Hoeting. (1997). "Bayesian Model Averaging for Linear Regression Models," Journal of the American Statistical Association 92, 179-191.

Raftery, A.E., Painter, I. and Volinsky, C.T. (2005). "BMA: An R package for Bayesian Model Averaging." $R$ News 5, no. 2, 2-8.

Raftery, A.E., J.A. Hoeting, C.T. Volinsky, I. Painter and K.Y. Yeung (2009). "BMA: An R package for Bayesian Model Averaging." URL: http://cran.r-project.org/web/packages/BMA/

Sala-i-Martin, X. (1997). "I Just Ran Two Million Regressions," AEA Papers and Proceedings 87, 178-183.

Sala-i-Martin, X., G. Doppelhofer and R.I. Miller. (2004). "Determinants of Long-Term Growth: A Bayesian Averaging of Classical Estimates (BACE) Approach," American Economic Review 94, 813-835.

Schwarz. G. (1978). "Estimating the Dimension of a Model," Annals of Statistics 6, 461-464.

Taplin, R.H. and Raftery, A.E. (1994). "Analysis of agricultural field trials in the presence of outliers and fertility jumps." Biometrics 50, 764-781. 
Wasserman, L. (2000). "Bayesian Model Selection and Model Averaging," Journal of Mathematical Psychology 44, 92-107.

Weigend, A.S., and S. Shi. (2000). "Predicting Daily Probability Distributions of S\&P500 Returns," Journal of Forecasting 19, 375-392.

Zellner, A. (1986). "On Assessing Prior Distributions and Bayesian Regression Analysis with $g$-prior Distributions," in Bayesian Inference and Decision Techniques: Essays in Honor of Bruno de Finetti. Goel, P.K. and A. Zellner, eds., North-Holland, Amsterdam. 
Table 1: Parameter Prior Structures

\begin{tabular}{|c|c|c|c|}
\hline Prior & Specification of g-prior & Comment & $\begin{array}{c}\text { Source } \\
\end{array}$ \\
\hline 1 & Unit Information Prior & $\begin{array}{l}\text { The prior contains information approximately equal to } \\
\text { that contained in a single typical observation. The } \\
\text { resulting posterior model probabilities are closely } \\
\text { approximated by the Schwarz Criterion, BIC. }\end{array}$ & $\begin{array}{l}\text { Kass and Wasserman (1995), } \\
\text { Raftery (1995) }\end{array}$ \\
\hline 2 & $g_{k}=p_{k} / n$ & $\begin{array}{l}\text { Prior information increases with the number of } \\
\text { regressors in the model. }\end{array}$ & FLS(2001b) \\
\hline 3 & $g_{k}=p^{1 / p_{k}} / n$ & $\begin{array}{l}\text { Prior information decreases with the number of } \\
\text { regressors in the model. }\end{array}$ & FLS(2001b) \\
\hline 4 & $g=1 / \sqrt{n}$ & $\begin{array}{l}\text { This is an intermediate case of prior } 1 \text { suggested by } \\
\text { FLS where a smaller asymptotic penalty is chosen for } \\
\text { larger models. }\end{array}$ & FLS(2001b) \\
\hline 5 & $g_{k}=\sqrt{p_{k} / n}$ & $\begin{array}{l}\text { This is an intermediate case of prior } 2 \text {, suggested by } \\
\text { FLS, where prior information increases with the } \\
\text { number of regressors in the model. }\end{array}$ & FLS(2001b) \\
\hline 6 & $g=1 /(\ln n)^{3}$ & $\begin{array}{l}\text { The Hannan-Quinn criterion. } \mathrm{CHQ}=3 \text { as } \mathrm{n} \text { becomes } \\
\text { large. }\end{array}$ & Hannan-Quinn (1979) \\
\hline 7 & $g_{k}=\ln \left(p_{k}+1\right) /(\ln n)$ & $\begin{array}{l}\text { Prior information decreases even slower with sample } \\
\text { size and there is asymptotic convergence to the } \\
\text { Hannan-Quinn criterion with } \mathrm{CHQ}=1 \text {. }\end{array}$ & Hannan-Quinn (1979) \\
\hline 8 & $g_{k}=\delta \gamma^{\left(1 / p_{k}\right)} /\left(1-\delta \gamma^{\left(1 / p_{k}\right)}\right)$ & $\begin{array}{l}\text { A natural conjugate prior structure, subjectively } \\
\text { elicited through predictive implications. } \gamma<1 \text { (so that } \\
\mathrm{g} \text { increases with } k_{j} \text { ) and delta such that } \mathrm{g} /(1+\mathrm{g}) \in \\
{[0.10,0.15] \text { (the weight of the "prior prediction error" }} \\
\text { in the Bayes factors); for } k_{j} \text { ranging from } 1 \text { to } 15 \text {. FLS } \\
\text { suggest covering this interval with the values of } \gamma= \\
0.65 \text { and } \delta=0.15 \text {. }\end{array}$ & Laud and Ibrahim (1996) \\
\hline 9 & $g=1 / p^{2}$ & $\begin{array}{l}\text { This prior is suggested by the risk inflation criterion } \\
\text { (RIC). }\end{array}$ & Foster and George (1994) \\
\hline 10 & $g=1 /\left(\max \left[n, p^{2}\right]\right)$ & $\begin{array}{l}\text { The preferred prior of Fernandez Ley and Steel (2001), } \\
\text { a mix of Prior } 9 \text { and Prior } 1 \text {. }\end{array}$ & FLS (2001b) \\
\hline 11 & $\begin{array}{l}\beta \sim N\left(\mu, \sigma^{2} V\right) \\
V=\sigma^{2} \phi^{2}\left(1 / n X^{\prime} X\right)^{-1} \\
\nu \lambda / \sigma^{2} \sim \chi^{2}\end{array}$ & $\begin{array}{l}\text { Data dependent prior. } \phi=2.85, v=2.58, \lambda=0.28 \\
\text { if the } \mathrm{R}^{2} \text { of the full model is less than } 0.9 \text {, and } \phi= \\
9.2, v=0.2, \lambda=0.1684 \text { if the } \mathrm{R}^{2} \text { of the full model is } \\
\text { greater than } 0.9 \text {. }\end{array}$ & $\begin{array}{l}\text { Raftery, Madigan and } \\
\text { Hoeting (1997) }\end{array}$ \\
\hline 12 & $g=n^{-1}$ & $\begin{array}{l}\text { Similar to the Unit Information Prior, but with mean } \\
\text { zero instead of MLE. }\end{array}$ & FLS(2001b) \\
\hline
\end{tabular}


Table 2

Posterior Inclusion Probabilities Across Parameter Priors

Model Prior $=$ Uniform

(Growth Dataset)

\begin{tabular}{|c|c|c|c|c|c|c|c|c|c|c|c|}
\hline & \multicolumn{11}{|c|}{ Priors Arranged By Effective g-Value (increasing left to right) } \\
\hline & Prior 11 & 9 (FLS) & Prior 6 & Prior 1 & Prior 12 & Prior 3 & Prior 4 & Prior 8 & Prior 2 & Prior 5 & Prior 7 \\
\hline Confucius & 99.5 & 99.9 & 100.0 & 100.0 & 100.0 & 100.0 & 100.0 & 100.0 & 99.9 & 99.2 & 98.5 \\
\hline GDPsh560 & 99.9 & 99.9 & 100.0 & 100.0 & 100.0 & 100.0 & 100.0 & 100.0 & 100.0 & 99.5 & 98.5 \\
\hline Life & 96.5 & 96.4 & 99.9 & 100.0 & 100.0 & 99.9 & 99.8 & 98.6 & 96.4 & 93.1 & 90.9 \\
\hline RuleofLaw & 47.2 & 64.0 & 99.6 & 100.0 & 99.6 & 99.6 & 98.3 & 93.0 & 69.3 & 57.3 & 56.6 \\
\hline SubSahara & 74.8 & 83.8 & 99.9 & 100.0 & 100.0 & 100.0 & 99.7 & 97.5 & 86.3 & 80.2 & 79.6 \\
\hline EquipInv & 99.0 & 96.8 & 98.3 & 99.9 & 98.4 & 98.3 & 95.6 & 88.8 & 94.4 & 95.3 & 95.2 \\
\hline Hindu & 3.2 & 10.3 & 96.6 & 99.9 & 97.0 & 96.8 & 88.7 & 42.8 & 16.7 & 15.0 & 18.5 \\
\hline HighEnroll & 0.3 & 0.7 & 93.4 & 99.8 & 94.0 & 93.5 & 78.1 & 2.8 & 2.1 & 3.9 & 7.2 \\
\hline LabForce & 0.4 & 1.3 & 94.5 & 99.8 & 95.0 & 94.6 & 81.6 & 11.6 & 3.9 & 5.6 & 9.2 \\
\hline EthnoLFrac & 0.5 & 1.3 & 90.8 & 99.3 & 91.4 & 90.8 & 74.6 & 7.2 & 3.3 & 4.8 & 8.0 \\
\hline Mining & 28.0 & 38.5 & 96.4 & 99.2 & 96.5 & 96.4 & 93.3 & 74.7 & 49.1 & 43.4 & 44.1 \\
\hline LatAmerica & 9.2 & 13.4 & 79.5 & 97.2 & 80.3 & 79.4 & 61.0 & 30.2 & 17.7 & 17.5 & 19.1 \\
\hline SpanishCol & 0.0 & 0.1 & 67.6 & 94.6 & 68.7 & 67.3 & 42.3 & 2.0 & 0.5 & 1.1 & 2.4 \\
\hline FrenchCol & 0.3 & 0.2 & 65.4 & 93.9 & 66.5 & 65.1 & 39.4 & 0.0 & 0.3 & 1.0 & 2.2 \\
\hline BritCol & 0.0 & 0.0 & 64.7 & 93.6 & 65.8 & 64.4 & 38.7 & 0.7 & 0.2 & 0.6 & 1.8 \\
\hline PrSc & 19.3 & 12.0 & 72.2 & 90.7 & 72.8 & 72.2 & 58.0 & 8.1 & 14.1 & 16.1 & 17.5 \\
\hline CivlLib & 5.2 & 3.3 & 66.8 & 85.7 & 67.5 & 66.7 & 51.2 & 3.7 & 4.4 & 5.4 & 7.1 \\
\hline NEquipInv & 28.8 & 49.3 & 71.3 & 85.6 & 71.7 & 71.3 & 66.6 & 82.1 & 52.4 & 41.1 & 40.3 \\
\hline English. & 0.5 & 1.1 & 58.0 & 84.5 & 58.9 & 57.7 & 36.7 & 2.7 & 2.2 & 2.4 & 3.5 \\
\hline OutwarOr & 0.0 & 0.0 & 51.2 & 82.8 & 52.2 & 51.0 & 31.4 & 0.7 & 0.2 & 0.6 & 1.7 \\
\hline BlMktPm & 5.1 & 12.2 & 63.8 & 72.5 & 63.9 & 64.1 & 67.6 & 45.4 & 19.6 & 17.4 & 19.9 \\
\hline Muslim & 66.9 & 68.3 & 44.3 & 60.9 & 44.4 & 44.4 & 49.4 & 54.9 & 66.5 & 60.3 & 56.1 \\
\hline Buddha & 4.1 & 10.2 & 19.5 & 36.5 & 19.7 & 19.7 & 21.5 & 31.1 & 13.4 & 10.6 & 11.4 \\
\hline EcoOrg & 34.2 & 56.6 & 39.5 & 35.6 & 39.2 & 39.7 & 50.1 & 88.7 & 61.0 & 47.3 & 45.2 \\
\hline X.PublEdu & 0.0 & 0.2 & 17.9 & 13.3 & 17.8 & 18.1 & 19.4 & 1.5 & 0.6 & 1.1 & 2.0 \\
\hline PolRights & 2.0 & 2.7 & 16.4 & 12.4 & 16.5 & 16.5 & 14.6 & 10.1 & 4.5 & 4.4 & 4.8 \\
\hline Protestants & 35.5 & 51.5 & 25.7 & 11.7 & 25.2 & 26.0 & 41.7 & 81.3 & 56.8 & 47.7 & 46.4 \\
\hline WarDummy & 1.1 & 0.9 & 6.2 & 11.7 & 6.4 & 6.3 & 3.9 & 0.8 & 1.2 & 1.8 & 2.0 \\
\hline Age & 0.4 & 0.7 & 14.6 & 11.4 & 14.7 & 14.7 & 12.2 & 3.3 & 1.3 & 1.7 & 2.3 \\
\hline RFEXDist & 1.8 & 2.0 & 4.6 & 9.6 & 4.7 & 4.7 & 4.0 & 0.6 & 2.6 & 3.3 & 3.4 \\
\hline Catholic & 4.1 & 8.7 & 3.5 & 7.5 & 3.5 & 3.6 & 7.1 & 20.3 & 11.0 & 8.3 & 8.2 \\
\hline Popg & 0.2 & 0.3 & 2.2 & 3.6 & 2.2 & 2.3 & 2.2 & 0.2 & 0.5 & 0.5 & 0.5 \\
\hline PrExports & 2.2 & 2.5 & 1.2 & 2.8 & 1.2 & 1.2 & 2.1 & 5.9 & 3.7 & 3.0 & 2.8 \\
\hline Foreign. & 0.5 & 0.3 & 0.7 & 2.0 & 0.7 & 0.7 & 0.4 & 0.0 & 0.2 & 0.6 & 0.7 \\
\hline Jewish & 0.0 & 0.0 & 0.8 & 1.3 & 0.8 & 0.8 & 0.7 & 0.0 & 0.0 & 0.0 & 0.1 \\
\hline std.BMP. & 0.0 & 0.0 & 0.6 & 1.3 & 0.6 & 0.6 & 0.4 & 0.0 & 0.0 & 0.0 & 0.0 \\
\hline Area & 0.0 & 0.0 & 0.8 & 1.1 & 0.9 & 0.9 & 1.1 & 0.0 & 0.1 & 0.1 & 0.2 \\
\hline Work.Pop & 0.4 & 0.2 & 0.3 & 1.1 & 0.3 & 0.3 & 0.2 & 0.0 & 0.2 & 0.6 & 0.8 \\
\hline AbsLat & 0.6 & 0.5 & 1.2 & 1.0 & 1.2 & 1.2 & 1.8 & 0.3 & 0.7 & 0.9 & 1.0 \\
\hline YrsOpen & 57.8 & 40.9 & 1.2 & 1.0 & 1.1 & 1.2 & 3.4 & 15.3 & 37.3 & 44.2 & 42.4 \\
\hline Rev.Coup & 0.1 & 0.2 & 0.4 & 0.7 & 0.4 & 0.4 & 0.7 & 1.1 & 0.5 & 0.4 & 0.4 \\
\hline $\begin{array}{l}\text { \# of relevant } \\
\text { regressors }\end{array}$ & 7 & 9 & 21 & 22 & 21 & 21 & 17 & 11 & 10 & 7 & 7 \\
\hline
\end{tabular}

1) Posterior inclusion probabilities that exceed $50 \%$ are in bold font (Jeffreys, 1961)

2) Priors 9 and 10 are identical in the growth context 
Table 3

Posterior Inclusion Probabilities Across Parameter and Model Priors

Uniform Model Prior Column 1, All Other Columns: Prior Model Size =7 (as in Sala-i-Martin et al., 2004)

(Growth Dataset)

\begin{tabular}{|c|c|c|c|c|c|c|c|c|c|c|c|c|}
\hline & \multirow{2}{*}{$\begin{array}{c}\text { Prior } 1 \\
\text { Model Prior: } \\
\text { Uniform } \\
\end{array}$} & \multicolumn{11}{|c|}{ Priors Arranged By Effective g-Value (increasing left to right) } \\
\hline & & Prior 11 & Prior 9 & Prior 6 & Prior 1 & Prior 12 & Prior 3 & Prior 4 & Prior 8 & Prior 2 & Prior 5 & Prior 7 \\
\hline Confucius & 100.0 & 92.0 & 95.8 & 99.7 & 99.9 & 99.7 & 99.7 & 98.7 & 97.2 & 96.5 & 87.1 & 84.8 \\
\hline GDPsh560 & 100.0 & 91.6 & 91.7 & 99.8 & 100.0 & 99.8 & 99.8 & 99.0 & 97.3 & 96.8 & 71.8 & 50.1 \\
\hline Life & 100.0 & 79.5 & 77.4 & 94.8 & 97.8 & 94.9 & 94.8 & 90.2 & 84.9 & 82.0 & 48.8 & 30.8 \\
\hline RuleofLaw & 100.0 & 16.5 & 16.9 & 49.4 & 68.6 & 50.2 & 50.4 & 37.0 & 29.2 & 21.5 & 12.3 & 8.2 \\
\hline SubSahara & 100.0 & 61.8 & 60.4 & 76.5 & 86.3 & 76.9 & 77.0 & 70.1 & 66.1 & 62.9 & 48.5 & 35.1 \\
\hline EquipInv & 99.9 & 99.5 & 99.4 & 98.2 & 99.2 & 98.1 & 98.0 & 98.5 & 98.7 & 99.0 & 98.5 & 97.9 \\
\hline Hindu & 99.9 & 0.0 & 0.0 & 4.8 & 9.6 & 5.0 & 5.1 & 2.3 & 1.1 & 0.1 & 0.0 & 0.0 \\
\hline HighEnroll & 99.8 & 0.1 & 0.1 & 0.1 & 1.0 & 0.1 & 0.1 & 0.1 & 0.1 & 0.1 & 0.8 & 1.2 \\
\hline LabForce & 99.8 & 0.0 & 0.0 & 0.3 & 1.5 & 0.3 & 0.3 & 0.1 & 0.0 & 0.0 & 0.0 & 0.0 \\
\hline EthnoLFrac & 99.3 & 0.2 & 0.2 & 0.4 & 0.9 & 0.5 & 0.5 & 0.4 & 0.4 & 0.4 & 0.5 & 0.3 \\
\hline Mining & 99.2 & 4.1 & 6.9 & 31.2 & 33.7 & 31.8 & 32.2 & 25.8 & 19.6 & 12.0 & 3.8 & 1.7 \\
\hline LatAmerica & 97.2 & 4.7 & 6.0 & 11.2 & 11.1 & 11.4 & 11.6 & 11.6 & 10.9 & 9.3 & 6.1 & 3.9 \\
\hline SpanishCol & 94.6 & 0.0 & 0.0 & 0 & 0.0 & 0.0 & 0.0 & 0.0 & 0.0 & 0.0 & 0.0 & 0.0 \\
\hline FrenchCol & 93.9 & 0.3 & 0.3 & 0.3 & 0.0 & 0.3 & 0.3 & 0.6 & 0.7 & 0.7 & 0.3 & 0.1 \\
\hline BritCol & 93.6 & 0.0 & 0.0 & 0 & 0.0 & 0.0 & 0.0 & 0.0 & 0.0 & 0.0 & 0.0 & 0.0 \\
\hline PrSc & 90.7 & 6.6 & 7.8 & 13.3 & 8.0 & 13.3 & 13.5 & 14.6 & 13.6 & 11.5 & 6.5 & 4.8 \\
\hline CivlLib & 85.7 & 1.0 & 1.2 & 3.2 & 2.2 & 3.2 & 3.3 & 3.3 & 2.9 & 2.1 & 0.6 & 0.4 \\
\hline NEquipInv & 85.6 & 3.2 & 5.6 & 34.7 & 56.2 & 35.4 & 35.5 & 23.0 & 16.6 & 9.8 & 5.2 & 4.1 \\
\hline English. & 84.5 & 0.0 & 0.0 & 0.8 & 0.1 & 0.8 & 0.9 & 0.7 & 0.4 & 0.1 & 0.1 & 0.3 \\
\hline OutwarOr & 82.8 & 0.0 & 0.0 & 0 & 0.1 & 0.0 & 0.0 & 0.0 & 0.0 & 0.0 & 0.1 & 0.3 \\
\hline BlMktPm & 72.5 & 0.1 & 0.3 & 6.8 & 10.0 & 7.1 & 7.3 & 4.6 & 2.7 & 0.8 & 0.1 & 0.0 \\
\hline Muslim & 60.9 & 21.5 & 29.2 & 65.6 & 69.1 & 65.9 & 65.8 & 56.5 & 46.9 & 37.2 & 13.0 & 7.2 \\
\hline Buddha & 36.5 & 2.3 & 2.6 & 5.9 & 11.8 & 6.1 & 6.2 & 3.8 & 3.1 & 2.0 & 9.6 & 13.8 \\
\hline EcoOrg & 35.6 & 4.7 & 7.6 & 40.7 & 61.9 & 41.6 & 41.7 & 27.4 & 19.7 & 11.9 & 6.2 & 5.0 \\
\hline X.PublEdu & 13.3 & 0.0 & 0.0 & 0 & 0.2 & 0.0 & 0.0 & 0.0 & 0.0 & 0.0 & 0.0 & 0.0 \\
\hline PolRights & 12.4 & 0.3 & 0.5 & 1.9 & 0.8 & 1.9 & 2.0 & 2.0 & 1.7 & 1.2 & 0.4 & 0.5 \\
\hline Protestants & 11.7 & 16.8 & 21.3 & 40.7 & 51.8 & 41.3 & 41.5 & 32.6 & 27.4 & 21.4 & 24.9 & 25.6 \\
\hline WarDummy & 11.7 & 0.8 & 0.9 & 1.2 & 0.0 & 1.2 & 1.2 & 1.9 & 2.1 & 1.9 & 1.3 & 0.7 \\
\hline Age & 11.4 & 0.4 & 0.6 & 0.6 & 0.1 & 0.6 & 0.7 & 0.9 & 1.1 & 1.0 & 1.8 & 2.0 \\
\hline RFEXDist & 9.6 & 1.2 & 1.6 & 2.5 & 0.0 & 2.5 & 2.6 & 3.3 & 3.3 & 2.6 & 3.8 & 4.8 \\
\hline Catholic & 7.5 & 0.6 & 1.1 & 5.3 & 9.0 & 5.5 & 5.5 & 3.3 & 2.3 & 1.4 & 1.9 & 1.6 \\
\hline Popg & 3.6 & 0.0 & 0.0 & 0.2 & 0.0 & 0.2 & 0.3 & 0.2 & 0.1 & 0.0 & 0.1 & 0.2 \\
\hline PrExports & 2.8 & 0.1 & 0.1 & 1.8 & 1.3 & 1.9 & 1.9 & 1.4 & 0.9 & 0.3 & 0.5 & 0.5 \\
\hline Foreign. & 2.0 & 0.6 & 0.9 & 0.6 & 0.0 & 0.6 & 0.6 & 1.1 & 1.3 & 1.5 & 1.0 & 0.7 \\
\hline Jewish & 1.3 & 0.0 & 0.0 & 0 & 0.0 & 0.0 & 0.0 & 0.0 & 0.0 & 0.0 & 0.1 & 0.2 \\
\hline std.BMP. & 1.3 & 0.0 & 0.0 & 0 & 0.0 & 0.0 & 0.0 & 0.0 & 0.0 & 0.0 & 0.4 & 0.8 \\
\hline Area & 1.1 & 0.0 & 0.0 & 0 & 0.0 & 0.0 & 0.0 & 0.0 & 0.0 & 0.0 & 0.1 & 0.3 \\
\hline Work.Pop & 1.1 & 1.1 & 1.2 & 0.5 & 0.1 & 0.4 & 0.5 & 1.0 & 1.5 & 1.7 & 2.2 & 2.2 \\
\hline AbsLat & 1.0 & 0.2 & 0.3 & 0.6 & 0.0 & 0.6 & 0.6 & 0.8 & 0.8 & 0.7 & 0.2 & 1.0 \\
\hline YrsOpen & 1.0 & 59.8 & 63.0 & 52.4 & 38.0 & 51.8 & 51.7 & 59.2 & 61.0 & 63.5 & 49.1 & 38.2 \\
\hline Rev.Coup & 0.7 & 0.0 & 0.0 & 0.1 & 0.0 & 0.1 & 0.1 & 0.1 & 0.0 & 0.0 & 0.0 & 0.0 \\
\hline relevant regressors & 22 & 6 & 6 & 7 & 10 & 8 & 8 & 7 & 6 & 6 & 3 & 3 \\
\hline
\end{tabular}

1) Posterior inclusion probabilities that exceed $50 \%$ are in bold font (Jeffreys, 1961)

2) Priors 9 and 10 are identical in the growth context

3) Priors arranged by effective g-value (see Figure 1) 
Table 4a

Parameter Priors And Predictive Performance Performance Scores Relative to Parameter Prior 1 (Growth Dataset, Model Prior: Uniform) Subsamples: 400

\begin{tabular}{|clrl|}
\hline Prior & Mean $^{\mathbf{a}}$ & Median $^{\mathbf{a}}$ & \multicolumn{1}{c|}{$\mathbf{\%}^{\mathbf{b}}$} \\
\multicolumn{5}{|c}{ MSE } \\
$\mathbf{1 1}$ & 0.073 & 0.014 & $69^{* * *}$ \\
$\mathbf{9}$ & 0.075 & 0.012 & $69^{* * *}$ \\
$\mathbf{6}$ & 0.039 & 0.002 & $55^{* *}$ \\
$\mathbf{1 2}$ & 0.085 & 0.006 & $71^{* * *}$ \\
$\mathbf{3}$ & 0.083 & 0.005 & $69^{* * *}$ \\
$\mathbf{4}$ & 0.059 & 0.003 & $57^{* * *}$ \\
$\mathbf{8}$ & 0.051 & 0.003 & $58^{* * *}$ \\
$\mathbf{2}$ & 0.022 & 0.003 & $58^{* * *}$ \\
$\mathbf{5}$ & 0.008 & 0.003 & $55^{* *}$ \\
$\mathbf{7}$ & 0.013 & 0.004 & $56^{* * *}$ \\
\multicolumn{5}{c}{ CRPS } & \\
$\mathbf{1 1}$ & 0.854 & 0.030 & $69^{* * *}$ \\
$\mathbf{9}$ & 0.944 & 0.029 & $69^{* * *}$ \\
$\mathbf{6}$ & 0.233 & 0.005 & $57^{* * *}$ \\
$\mathbf{1 2}$ & 0.675 & 0.009 & $65^{* * *}$ \\
$\mathbf{3}$ & 0.533 & 0.007 & $65^{* * *}$ \\
$\mathbf{4}$ & 0.000 & 0.002 & 53 \\
$\mathbf{8}$ & 0.058 & 0.003 & $55^{* *}$ \\
$\mathbf{2}$ & 0.193 & 0.008 & $58^{* * *}$ \\
$\mathbf{5}$ & 0.708 & 0.012 & $60^{* * *}$ \\
$\mathbf{7}$ & 1.085 & 0.018 & $64^{* * *}$ \\
$\mathbf{1 1}$ & 0.711 & 0.711 & $61^{* * *}$ \\
$\mathbf{9}$ & 1.078 & 1.437 & $63^{* * *}$ \\
$\mathbf{6}$ & -1.617 & -0.715 & $41^{* * *}$ \\
$\mathbf{1 2}$ & 1.719 & 1.668 & $77^{* * *}$ \\
$\mathbf{3}$ & 1.337 & 1.348 & $73^{* * *}$ \\
$\mathbf{4}$ & -1.557 & -0.780 & $38^{* * *}$ \\
$\mathbf{8}$ & -1.647 & -0.846 & $39^{* * *}$ \\
$\mathbf{2}$ & -1.181 & -0.435 & $44^{* * *}$ \\
$\mathbf{5}$ & -0.755 & 0.178 & 51 \\
$\mathbf{7}$ & -0.250 & 0.731 & $56^{* * *}$ \\
\hline
\end{tabular}

${ }^{a}$ Refers to the improvement in the score attained by the UIP compared to a given alternative prior

b Indicates percent of trials where "success" is a better predictive score by the UIP than by the alternative prior

$* * *, * *, *$ are $99 \%, 95 \%, 90 \%$ one-sided significance levels based on binomial $\mathrm{p}$ values, $\mathrm{P}(\mathrm{X}>\mathrm{or}=\mathrm{z})$, for the given number of trials and successes; where success is defined as a better score for prior 1 (the alternative) as compared to the alternative prior (UIP) if the percentage is above (below) $50 \%$

1) Priors 9 and 10 are identical in the growth context 2) Priors arranged by effective g-value (see Figure 1)
Table 4b

Priors And Predictive Performance: Comparison to Ley and Steel (2007b) Absolute Performance Scores, Log Predictive Score (Growth Dataset, Model Prior: Uniform) Subsamples: 100

\begin{tabular}{|c|c|c|c|c|c|}
\hline \multirow{3}{*}{\begin{tabular}{|l|} 
model prior \\
autors \\
parameter prior \\
\end{tabular}} & \multicolumn{3}{|c|}{ fixed, uniform } & \multirow{2}{*}{\multicolumn{2}{|c|}{$\begin{array}{c}\text { random } \\
\text { LS } \\
\end{array}$}} \\
\hline & EPR & \multicolumn{2}{|c|}{ LS } & & \\
\hline & 1 & 9 & 12 & 9 & 12 \\
\hline $\min$ & 0.16 & 1.11 & 0.86 & 1.20 & 1.11 \\
\hline mean & 0.97 & 1.63 & 1.65 & 1.63 & 1.61 \\
\hline $\max$ & 2.32 & 2.85 & 2.76 & 2.47 & 2.64 \\
\hline stdev & 0.47 & 0.37 & 0.42 & 0.25 & 0.34 \\
\hline
\end{tabular}

100 random split trials (subsamples) and $15 \%$ hold out sample

LS: Ley and Steel (2007b)

EPR: Eicher, Papageorgiou, Raftery

Log Predictive Score: to conform to the Ley and Steel's LPS definition we divide here by the number of held out regressors 
Table 5

Parameter Priors, Model Priors, and Predictive Performance (Growth Dataset)

Performance Scores Relative to Prior 1 with Uniform Model Prior

$$
\text { Subsamples }=190
$$

\begin{tabular}{|c|c|c|c|c|c|c|c|c|c|c|c|c|c|c|c|c|c|c|c|c|}
\hline \multirow{3}{*}{ Prior } & \multicolumn{2}{|c|}{ Prior Model Size=3 } & \multirow{2}{*}{\multicolumn{2}{|c|}{ Prior Model Size=5 }} & \multirow{2}{*}{\multicolumn{2}{|c|}{ Prior Model Size $=6$}} & \multirow{2}{*}{\multicolumn{2}{|c|}{ Prior Model Size $=7$}} & \multicolumn{2}{|c|}{ Prior Model Size $=\mathbf{8}$} & \multirow{2}{*}{\multicolumn{2}{|c|}{ Prior Model Size $=9$}} & \multicolumn{2}{|c|}{ Prior Model Size=11 } & \multicolumn{2}{|c|}{ Prior Model Size $=13$} & \multicolumn{2}{|c|}{ Prior Model Size $=15$} & \multicolumn{2}{|c|}{ Prior Model Size=17 } \\
\hline & Median $^{\mathrm{a}}$ & $\%^{c}$ & & & & & & & Median $^{a}$ & & & & Median $^{a}$ & & Median $^{2}$ & & Median & & Median $^{a}$ & \\
\hline & \multicolumn{2}{|c|}{ MSE } & \multicolumn{2}{|c|}{ MSE } & \multicolumn{2}{|c|}{ MSE } & \multicolumn{2}{|c|}{ MSE } & \multicolumn{2}{|c|}{ MSE } & \multicolumn{2}{|c|}{ MSE } & \multicolumn{2}{|c|}{ MSE } & \multicolumn{2}{|c|}{ MSE } & \multicolumn{2}{|c|}{ MSE } & & MSE \\
\hline 11 & 0.16 & & 0.13 & $72 * * *$ & 0.14 & & 0.13 & $71^{* * * *}$ & 0.12 & $71 * * *$ & 0.16 & $79 * * *$ & 0.17 & $81 * * *$ & 0.11 & $71^{* * * *}$ & 0.10 & $71 * * *$ & 0.10 & $72^{* * *}$ \\
\hline 9 & 0.15 & $71^{* * *}$ & 0.12 & $71^{* * * *}$ & 0.14 & $78 * * *$ & 0.12 & $71^{* * *}$ & 0.12 & $71^{* * * *}$ & 0.16 & $81^{* * *}$ & 0.16 & $80^{* * *}$ & 0.11 & $73^{* * * *}$ & 0.11 & $74^{* * * *}$ & 0.10 & $73^{* * *}$ \\
\hline 6 & 0.09 & $70^{* * *}$ & 0.08 & $71^{* * * *}$ & 0.13 & $75^{* * *}$ & 0.09 & $73^{* * *}$ & 0.09 & $72^{* * *}$ & 0.15 & $79^{* * *}$ & 0.12 & $77^{* * * *}$ & 0.08 & $80^{* * * *}$ & 0.08 & $81^{* * * *}$ & 0.07 & $79 * * *$ \\
\hline 1 & 0.03 & $67 * * *$ & 0.02 & $67 * * *$ & 0.02 & $67^{* * * *}$ & 0.02 & $69^{* * *}$ & 0.02 & $68^{* * *}$ & 0.04 & $58 * * *$ & 0.02 & $69^{* * * *}$ & 0.01 & $68^{* * * *}$ & 0.01 & $69 * * *$ & 0.01 & $70^{* * *}$ \\
\hline 12 & 0.10 & $70^{* * *}$ & 0.09 & $71^{* * * *}$ & 0.13 & $75^{* * * *}$ & 0.09 & $72^{* * *}$ & 0.09 & $73^{* * *}$ & 0.15 & $80^{* * *}$ & 0.12 & $78^{* * * *}$ & 0.08 & $82^{* * * *}$ & 0.08 & $81^{* * * *}$ & 0.07 & $81^{* * *}$ \\
\hline 3 & 0.09 & $70^{* * *}$ & 0.08 & $71^{* * * *}$ & 0.13 & $75^{* * *}$ & 0.09 & $73^{* * *}$ & 0.09 & $72^{* * *}$ & 0.15 & $79^{* * *}$ & 0.12 & $77^{* * * *}$ & 0.08 & $80^{* * * *}$ & 0.08 & $81^{* * *}$ & 0.07 & $79^{* * * *}$ \\
\hline 4 & 0.08 & $68^{* * * *}$ & 0.06 & $67 * * *$ & 0.09 & $70^{* * *}$ & 0.05 & $65^{* * * *}$ & 0.05 & $65^{* * *}$ & 0.13 & $78^{* * *}$ & 0.11 & $76^{* * * *}$ & 0.06 & $69^{* * * *}$ & 0.06 & $73^{* * *}$ & 0.05 & $73^{* * *}$ \\
\hline 8 & 0.09 & $67^{* * *}$ & 0.08 & $65^{* * * *}$ & 0.09 & $68^{* * *}$ & 0.05 & $65^{* * *}$ & 0.05 & $65^{* * *}$ & 0.13 & $74^{* * * *}$ & 0.12 & $76^{* * * *}$ & 0.05 & $66 * * *$ & 0.05 & $71^{* * *}$ & 0.05 & $71^{* * * *}$ \\
\hline 2 & 0.09 & $67^{* * * *}$ & 0.07 & $68^{* * * *}$ & 0.10 & $70^{* * * *}$ & 0.07 & $68^{* * * *}$ & 0.06 & $67 * * *$ & 0.12 & $74^{* * *}$ & 0.14 & $76^{* * * *}$ & 0.05 & $63^{* * *}$ & 0.06 & $65^{* * * *}$ & 0.05 & $65^{* * * *}$ \\
\hline 5 & 0.15 & $70^{* * *}$ & 0.13 & $69 * * *$ & & $76^{* * * *}$ & 0.11 & $67^{* * * *}$ & 0.11 & $67^{* * * *}$ & 0.17 & $73^{* * *}$ & 0.17 & $77^{* * * *}$ & 0.09 & $65^{* * * *}$ & 0.09 & $64 * * *$ & 0.08 & $65^{* * * *}$ \\
\hline 7 & 0.19 & $\begin{array}{l}75^{* * * *} \\
\text { RPS }\end{array}$ & & $\begin{array}{l}73 * * * \\
\text { CRPS }\end{array}$ & 0.18 & $\begin{array}{l}80^{* * * *} \\
\mathrm{RPS}\end{array}$ & 0.16 & $\begin{array}{l}73 * * * \\
\mathrm{RPS}\end{array}$ & 0.15 & $\begin{array}{l}72 * * * \\
\text { RPS }\end{array}$ & 0.20 & $\begin{array}{l}75^{* * *} \\
\mathrm{RPS}\end{array}$ & 0.20 & $\begin{array}{l}80 * * * \\
\mathrm{RPS}\end{array}$ & 0.13 & $\begin{array}{l}69 * * * \\
\text { RPS }\end{array}$ & 0.12 & $\begin{array}{l}69 * * * \\
\text { CRPS }\end{array}$ & 0.10 & $\begin{array}{l}68 * * * \\
\text { CRPS }\end{array}$ \\
\hline 11 & 0.04 & & 0.04 & & 0.03 & & & & 0.02 & $77 * * *$ & 0.03 & & & $78^{* * * *}$ & & $77^{* * *}$ & 0.01 & & 0.01 & \\
\hline 9 & 0.04 & $85^{* * *}$ & 0.03 & $77 * * *$ & 0.02 & $79 * * *$ & 0.02 & $79 * * *$ & 0.022 & $75^{* * *}$ & 0.02 & $71^{* * * *}$ & 0.04 & $75^{* * * *}$ & 0.01 & $73^{* * * *}$ & 0.01 & $74 * * *$ & 0.01 & $71^{* * * *}$ \\
\hline 6 & 0.01 & $69^{* * *}$ & 0.01 & $66^{* * *}$ & 0.01 & $69^{* * *}$ & 0.01 & $66^{* * * *}$ & 0.01 & $67 * * *$ & 0.01 & $62^{* * *}$ & 0.01 & $63^{* * *}$ & 0.00 & $71^{* * * *}$ & 0.00 & $62^{* * * *}$ & 0.00 & $63^{* * *}$ \\
\hline 1 & 0.00 & $61^{* * * *}$ & 0.00 & $59 * *$ & 0.00 & $62^{* * *}$ & 0.00 & $61 * * *$ & 0.00 & $61^{* * * *}$ & 0.01 & 53 & 0.00 & $59 * *$ & 0.00 & $57^{* * *}$ & 0.00 & $57 * *$ & 0.00 & $55 * *$ \\
\hline 12 & 0.02 & $73^{* * * *}$ & 0.01 & $68^{* * * *}$ & 0.01 & $71^{* * * *}$ & 0.01 & $71^{* * * *}$ & 0.01 & $69 * * *$ & 0.01 & $62^{* * * *}$ & 0.01 & $63^{* * *}$ & 0.00 & $68 * * *$ & 0.00 & $65 * * *$ & 0.00 & $68 * * *$ \\
\hline 3 & 0.01 & $69^{* * *}$ & 0.01 & $66^{* * * *}$ & 0.01 & $69^{* * * *}$ & 0.01 & $66^{* * * *}$ & 0.01 & $67 * * *$ & 0.01 & $62^{* * * *}$ & 0.01 & $63 * * *$ & 000 & $77^{2 * * *}$ & 0.00 & $62 * * *$ & 000 & $63 * * *$ \\
\hline 4 & 0.01 & $67 * * *$ & 0.00 & $56^{*}$ & 0.00 & $59 * *$ & 0.00 & $56^{*}$ & 000 & 53 & 0.01 & $59 * *$ & 0.00 & 53 & 0.00 & $59 * *$ & 0.00 & $57 * *$ & 0.00 & $57 * *$ \\
\hline 8 & 0.01 & $65^{* * *}$ & 0.00 & $57 * *$ & 0.00 & $59 * *$ & 0.00 & $56^{*}$ & 000 & $56 *$ & 000 & $55 * * *$ & 0.00 & $56^{* * *}$ & 0.00 & 53 & 000 & 55 & 000 & $56^{*}$ \\
\hline 2 & 0.01 & $72^{* * *}$ & 001 & $66^{* * * *}$ & 0.01 & $66 * * *$ & 0.00 & $58^{* * *}$ & 000 & $57 * *$ & 001 & $61 * * *$ & 0.01 & $65 * * *$ & 0.00 & 53 & 000 & 54 & 000 & 52 \\
\hline 5 & 0.01 & $65^{* * * *}$ & 0.01 & $65 * * *$ & 0.00 & $63^{* * *}$ & 0.00 & $61^{* * * *}$ & 0.00 & $66^{* * * *}$ & 0.01 & $59^{* * *}$ & 0.01 & $57^{* * * *}$ & 0.00 & 55 & 0.00 & 51 & 0.00 & 51 \\
\hline 7 & & $63^{* * *}$ & 0.01 & $58^{* *}$ & 0.00 & $60^{* * *}$ & 0.00 & $59 * *$ & & $62^{* * * *}$ & 0.01 & $60^{* * * *}$ & 0.01 & $57 * * *$ & 0.00 & $55^{*}$ & 0.00 & 52 & 0.00 & 51 \\
\hline & & LPS & & LPS & & PS & & LPS & & LPS & & PS & & LPS & & LPS & & LPS & & LPS \\
\hline 11 & 1.50 & $62^{* * * *}$ & 1.17 & $59^{* *}$ & 1.89 & $64 * * *$ & 1.23 & $58^{* * *}$ & 1.15 & $57 * *$ & 3.58 & $82^{* * *}$ & 4.18 & $78^{* * *}$ & 0.82 & $55^{*}$ & 0.89 & $57^{* * *}$ & 0.99 & $57 * *$ \\
\hline 9 & 1.53 & $62^{* * * *}$ & 1.31 & $60^{* * * *}$ & 1.96 & $64 * * *$ & 1.30 & $59^{* *}$ & 1.16 & $59^{* *}$ & 3.51 & $82^{* * *}$ & 4.28 & $78 * * *$ & 1.10 & $56^{*}$ & 1.21 & $59 * *$ & 1.24 & $61^{* * * *}$ \\
\hline 6 & 0.40 & 54 & 0.66 & 54 & 1.72 & $59 * *$ & 0.68 & $55^{* * *}$ & 0.89 & 56 & 2.79 & $82^{* * *}$ & 3.23 & $75^{* * * *}$ & 1.26 & $65 * * *$ & 1.41 & $70^{* * *}$ & 1.60 & $72^{* * *}$ \\
\hline 1 & 0.86 & $62^{* * *}$ & 0.57 & $61^{* * * *}$ & 0.43 & $61 * * *$ & 0.34 & $60^{* * *}$ & 0.42 & $59^{* *}$ & 1.63 & $60^{* * *}$ & 0.32 & $61^{* * * *}$ & 0.22 & $61 * * *$ & 0.18 & $62^{* * *}$ & 0.10 & $63^{* * *}$ \\
\hline 12 & 0.66 & $56^{*}$ & 0.76 & 54 & 1.99 & $59 * *$ & 0.87 & $57 * *$ & 1.13 & $57^{* * *}$ & 2.89 & $83 * * *$ & 3.34 & $76^{* * *}$ & 1.46 & $67 * * *$ & 1.58 & $72^{* * *}$ & 1.94 & $73^{* * *}$ \\
\hline 3 & 0.40 & 54 & 0.66 & 54 & 1.72 & $59^{* * *}$ & 0.68 & 55 & 0.89 & $56^{* * *}$ & 2.79 & $82^{* * *}$ & 3.23 & $75^{* * *}$ & 1.26 & $65 * * *$ & 1.41 & $70^{* * *}$ & 1.60 & $72^{* * * *}$ \\
\hline 4 & 0.18 & 52 & -0.28 & 47 & 0.92 & $57^{* * *}$ & -0.44 & 47 & -0.44 & 47 & 2.36 & $77 * * *$ & 3.01 & $72^{* * *}$ & -0.54 & $44 * * *$ & -0.48 & $45^{*}$ & -0.53 & $43^{* *}$ \\
\hline 8 & 0.45 & 53 & -0.05 & 48 & 0.58 & $57^{* * *}$ & -0.43 & 47 & -0.40 & 47 & 2.37 & $77 * * *$ & 3.11 & $73^{* * *}$ & -0.62 & $42^{* * * *}$ & -0.61 & $43^{* * * *}$ & -0.82 & $44^{* *}$ \\
\hline 2 & 0.46 & 53 & 0.10 & 51 & 0.99 & $58 * *$ & -0.17 & 48 & -0.28 & 48 & 2.65 & $78 * * *$ & 3.29 & $75^{* * * *}$ & -0.39 & 48 & -0.40 & 46 & -0.54 & $45^{*}$ \\
\hline 5 & 1.45 & $59 * *$ & 1.11 & $58^{* * *}$ & 1.68 & $61^{* * *}$ & 0.81 & $56^{*}$ & 0.69 & $55^{*}$ & 3.43 & $88 * * *$ & 4.05 & $79 * * *$ & 0.20 & 52 & 0.02 & 50 & -0.02 & 50 \\
\hline 7 & 1.86 & $62^{* * * *}$ & 1.69 & $61^{* * *}$ & 2.06 & $64 * * *$ & 1.45 & $58^{* *}$ & 1.30 & $58^{* * *}$ & 4.22 & $91 * * *$ & 4.61 & $81^{* * * *}$ & 0.83 & $55^{*}$ & 0.69 & 54 & 0.57 & 53 \\
\hline
\end{tabular}

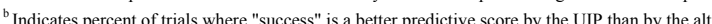
(the alternative) as compared to the alternative prior (UIP) if the percentage is above (below) $50 \%$

1) Priors 9 and 10 are identical in the growth context
2) Priors arranged by effective g-value (see Figure 1 ) 


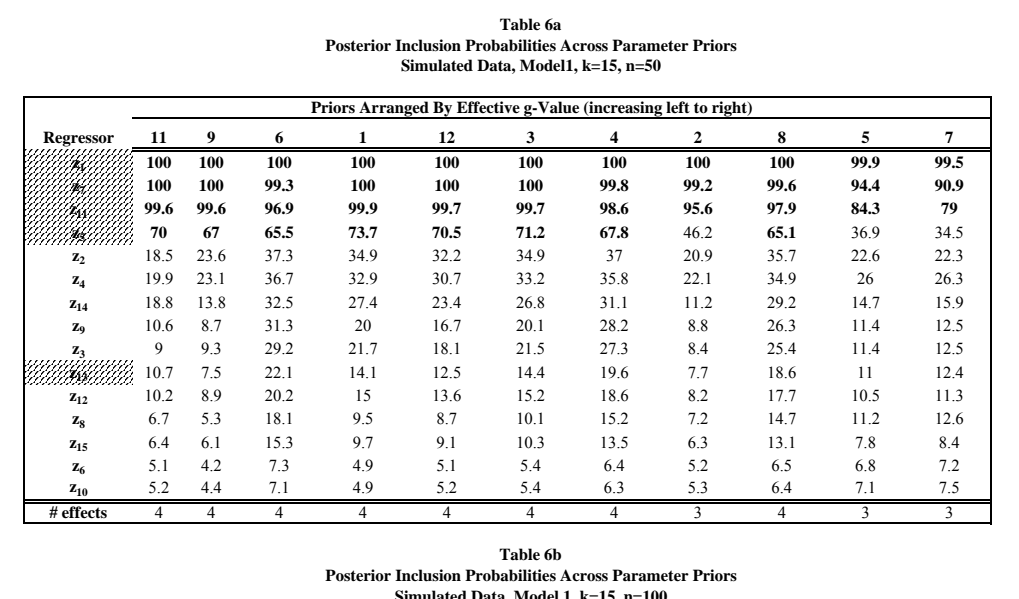

Simulated Data, Model 1, k=15, n=100

\begin{tabular}{|c|c|c|c|c|c|c|c|c|c|c|c|}
\hline \multirow[b]{2}{*}{ Regressor } & \multicolumn{11}{|c|}{ Priors Arranged By Effective g-Value (increasing left to right) } \\
\hline & 11 & 9 & 1 & 12 & 6 & 3 & 2 & 4 & 8 & 5 & 7 \\
\hline UIIIIIIIIIII & 100 & 100 & $\overline{100}$ & 100 & 100 & 100 & 100 & 100 & 100 & 100 & 100 \\
\hline $4 / /$ & 100 & 100 & 100 & 100 & 100 & 100 & 100 & 100 & 100 & 99.6 & 97.9 \\
\hline II & 99.4 & 99.4 & 99.7 & 99.5 & 99.5 & 99.5 & 97.6 & 99.1 & 98.1 & 86.5 & 75.6 \\
\hline $4=$ & 92.9 & 92.9 & 95.6 & 94.5 & 94.5 & 94.9 & 83.8 & 93.9 & 90.5 & 57.6 & 43.6 \\
\hline I & 79.9 & 81.1 & 87.8 & 85 & 85.1 & 86.2 & 63.2 & 85.1 & 78.8 & 35.8 & 28.3 \\
\hline $\mathbf{z}_{6}$ & 15.6 & 15.4 & 22.1 & 21.2 & 21.3 & 23.7 & 14.9 & 39 & 38 & 13 & 12.3 \\
\hline $\mathrm{z}_{12}$ & 13.7 & 13.2 & 19.2 & 18.3 & 18.4 & 20.5 & 12.4 & 33.2 & 32.2 & 10.9 & 10.4 \\
\hline $\mathbf{z}_{4}$ & 14.3 & 15.8 & 17.3 & 17.9 & 18 & 19.1 & 23 & 27.5 & 29.7 & 33.6 & 34.2 \\
\hline "YIXIIIIIIIII. & 7.7 & 6.9 & 9.9 & 9.7 & 9.7 & 10.9 & 7.1 & 16.7 & 16.6 & 7.9 & 8.8 \\
\hline $\mathrm{z}_{10}$ & 4.8 & 5.1 & 7.9 & 7.6 & 7.6 & 8.7 & 5.2 & 17.7 & 17.8 & 5.3 & 5.4 \\
\hline $\mathbf{z}_{3}$ & 4 & 6.1 & 7.4 & 7.6 & 7.6 & 8.3 & 7.7 & 12.3 & 13.1 & 9.1 & 8.7 \\
\hline $\mathbf{z}_{2}$ & 3.2 & 5 & 7 & 6.9 & 6.9 & 7.8 & 5.4 & 13.2 & 13.4 & 5.9 & 5.9 \\
\hline $\mathbf{z}_{8}$ & 6 & 5.6 & 7 & 7 & 7.1 & 7.7 & 6.4 & 11 & 11.3 & 7.4 & 7.7 \\
\hline$z_{9}$ & 4.9 & 4.6 & 6.8 & 6.6 & 6.7 & 7.6 & 4.9 & 14.3 & 14.4 & 5.2 & 5.2 \\
\hline $\mathrm{Z}_{14}$ & 4.6 & 4.3 & 6 & 5.9 & 6 & 6.7 & 4.6 & 10.9 & 11.1 & 5 & 5.3 \\
\hline \# effects & 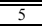 & $\begin{array}{l}5 \\
\end{array}$ & 5 & $\begin{array}{l}5 \\
\end{array}$ & 5 & 25 & $\begin{array}{l}5 \\
\end{array}$ & 25 & 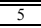 & 4 & 3 \\
\hline
\end{tabular}

Table 6c

Posterior Inclusion Probabilities Across Parameter Priors Simulated Data, Model 2, k=40, n=100

\begin{tabular}{|c|c|c|c|c|c|c|c|c|c|c|c|}
\hline \multirow[b]{2}{*}{ Regressor } & \multicolumn{11}{|c|}{ Priors Arranged By Effective g-Value (increasing left to right) } \\
\hline & 11 & 9 & 1 & 12 & $\begin{array}{c}6 \\
6\end{array}$ & 3 & $\begin{array}{c}4 \\
\end{array}$ & 8 & 2 & 5 & 7 \\
\hline $\mathrm{z}_{1}$ & 1.5 & 1.8 & 2.8 & 2.4 & 2 & 2.7 & 0.8 & 1.3 & 0.8 & 2.1 & 2 \\
\hline $\mathbf{z}_{2}$ & 0.9 & 1.2 & 8.6 & 1.7 & 1.5 & 2 & 0.2 & 0.1 & 0 & 0 & 0 \\
\hline $\mathbf{z}_{3}$ & 4.1 & 4.8 & 13.9 & 4.9 & 4.5 & 5.6 & 0.4 & 0.2 & 0 & 0.4 & 0.9 \\
\hline $\mathbf{z}_{4}$ & 0.6 & 0.6 & 1.6 & 1.1 & 1.3 & 1.2 & 0.1 & 0 & 0 & 1 & 2.1 \\
\hline$z_{5}$ & 0.3 & 0.4 & 1.9 & 0.8 & 0.5 & 0.9 & 0.2 & 0.6 & 0.7 & 0.2 & 0.1 \\
\hline $\mathbf{z}_{6}$ & 0.4 & 0.5 & 3.9 & 1 & 0.5 & 1.1 & 0.1 & 0 & 0 & 0 & 0 \\
\hline $\mathbf{z}_{7}$ & 0.3 & 0.3 & 1.5 & 0.8 & 0.1 & 0.9 & 0.2 & 0.5 & 0.9 & 0.5 & 0.3 \\
\hline$z_{8}$ & 0.4 & 0.6 & 4.5 & 1 & 0.1 & 1.1 & 0.1 & 0.1 & 0.1 & 1 & 1.1 \\
\hline $\mathbf{z}_{9}$ & 0.3 & 0.4 & 2.5 & 0.8 & 0.5 & 0.9 & 0.1 & 0 & 0 & 0 & 0 \\
\hline $\mathbf{z}_{10}$ & 0.4 & 0.4 & 1.6 & 0.9 & 0.6 & 0.9 & 0.1 & 0 & 0.1 & 1.3 & 1.9 \\
\hline $\mathrm{z}_{11}$ & 6.1 & 6.7 & 14.3 & 6.1 & 6.2 & 6.8 & 0.5 & 0.2 & 1.2 & 6.3 & 7 \\
\hline$z_{12}$ & 10.7 & 14.2 & 33.2 & 11.7 & 10.7 & 13.2 & 1.8 & 0.7 & 0 & 0 & 0 \\
\hline$z_{13}$ & 0.3 & 0.4 & 3 & 0.9 & 0.6 & 1 & 0.1 & 0 & 0 & 0 & 0.2 \\
\hline $\mathrm{z}_{14}$ & 12.7 & 12.6 & 6.8 & 15.7 & 14.7 & 16 & 12 & 7.8 & 0.5 & 0.4 & 0.2 \\
\hline$z_{15}$ & 0.4 & 0.5 & 3.9 & 0.9 & 0.1 & 1.1 & 0.1 & 0 & 0 & 0 & 0.1 \\
\hline $\mathrm{z}_{16}$ & 1.5 & 1.8 & 4.9 & 2.1 & 2.3 & 2.4 & 0.2 & 0.1 & 0 & 0.6 & 1.2 \\
\hline$z_{17}$ & 0.5 & 0.6 & 2.5 & 1 & 1 & 1.1 & 0.2 & 0.4 & 0.4 & 2.6 & 3.4 \\
\hline$z_{18}$ & 10.4 & 10.6 & 7.1 & 8.8 & 9.6 & 9.3 & 14.7 & 22.4 & 29.9 & 23.7 & 17.8 \\
\hline $\mathrm{z}_{19}$ & 0.8 & 1 & 6.1 & 1.4 & 1.3 & 1.7 & 1.4 & 3.6 & 9.3 & 10.6 & 9 \\
\hline$z_{20}$ & 0.6 & 0.7 & 2.7 & 1.2 & 1.4 & 1.3 & 1.7 & 1.5 & 1.9 & 1.2 & 1.2 \\
\hline YII & 4.4 & 7 & 57.1 & 4.2 & 4 & 5.3 & 0.4 & 0.9 & 2.1 & 1 & 0.6 \\
\hline . & 35.3 & 41.9 & 94 & 26.5 & 26.5 & 30 & 3.8 & 1.5 & 0 & 0.4 & 1.1 \\
\hline$y$ & 44.6 & 50.9 & 95.9 & 38.4 & 38.4 & 41.2 & 20.1 & 11.9 & 1.3 & 0.6 & 0.3 \\
\hline 4 & 98.7 & 99 & 100 & 93.3 & 93.2 & 93.7 & 38.2 & 19.8 & 0.5 & 3.8 & 5.3 \\
\hline I & 72.2 & 75.4 & 98.6 & 50.9 & 49.7 & 54.8 & 7.4 & 9.1 & 21.8 & 40.4 & 45.2 \\
\hline I & 99.7 & 99.8 & 100 & 96.8 & 96.6 & 97.1 & 29.1 & 14.7 & 1.1 & 1.1 & 0.8 \\
\hline I & 100 & 100 & 100 & 99.3 & 99.4 & 99.3 & 64.5 & 39.4 & 0.9 & 0.3 & 0.3 \\
\hline 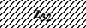 & 99 & 99.3 & 100 & 94.2 & 93.8 & 94.7 & 50.8 & 30.9 & 1.3 & 1 & 1.4 \\
\hline 1 & 100 & 100 & 100 & 100 & 100 & 100 & 72.5 & 45.7 & 2.6 & 2.7 & 2.9 \\
\hline I) & 100 & 100 & 100 & 100 & 100 & 100 & 81.9 & 56.9 & 3.1 & 2 & 2.3 \\
\hline 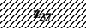 & 100 & 100 & 100 & 100 & 100 & 100 & 83.1 & 57.8 & 4.6 & 1.6 & 1.1 \\
\hline 4 & 100 & 100 & 100 & 100 & 100 & 100 & 97.3 & 86.7 & 31 & 13.4 & 10.4 \\
\hline 1 & 100 & 100 & 100 & 99.4 & 99.5 & 99.4 & 77.4 & 79 & 67.6 & 45.7 & 35.2 \\
\hline 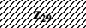 & 100 & 100 & 100 & 100 & 100 & 100 & 99.9 & 98.7 & 78.3 & 37.3 & 24.6 \\
\hline I & 100 & 100 & 100 & 100 & 100 & 100 & 99.4 & 95 & 55.7 & 26.6 & 19.9 \\
\hline - & 100 & 100 & 100 & 100 & 100 & 100 & 80.7 & 61.4 & 28.4 & 19.6 & 14 \\
\hline - & 100 & 100 & 100 & 100 & 100 & 100 & 99.9 & 99.2 & 90.2 & 64.5 & 50.5 \\
\hline II & 99 & 99.2 & 100 & 92.7 & 93.5 & 93.3 & 55.8 & 66.7 & 82.2 & 85.6 & 86.1 \\
\hline IIII! & 100 & 100 & 100 & 99.5 & 99.4 & 99.5 & 85.1 & 89.3 & 100 & 95.3 & 86.8 \\
\hline \# effects & 16 & 17 & 19 & 16 & 15 & 16 & 13 & 10 & 6 & 3 & 3 \\
\hline Black shad & & ss shoul & & & & & & & & & \\
\hline & & & & & & & & & & & \\
\hline & & & & & & & & & & & \\
\hline & & & & & & & & & & & \\
\hline
\end{tabular}


Table 7

Predictive Performance Relative to Parameter Prior 1 For The Three Simulated Datasets

Uniform Model Prior, 400 Subsamples

a) Model 1, k=15 n=50

b) Model 1, k=15 n=100

c) Model 2, $\mathrm{k}=40 \mathrm{n}=100$

\begin{tabular}{|c|c|c|c|c|c|c|c|c|c|c|}
\hline Prior & Mean $^{a}$ & $\begin{array}{c}\text { Median } \\
\text { MSE }\end{array}$ & $\%^{b}$ & Prior & Mean $^{a}$ & MSE & $\%^{b}$ & Prior & Mean $^{a}$ & $\operatorname{Median}^{\mathrm{a}} \quad \%^{\mathrm{b}}$ \\
\hline 11 & 0.004 & 0.003 & $55^{\star \star}$ & 11 & 0.114 & 0.120 & $70^{\star \star \star}$ & 11 & 0.010 & $0.00790^{* * *}$ \\
\hline 9 & 0.003 & 0.002 & $56^{\star *}$ & 9 & 0.127 & 0.127 & $71^{\star \star \star}$ & 9 & 0.007 & $0.00690^{\star \star \star}$ \\
\hline 6 & 0.029 & 0.026 & $67^{\star \star \star *}$ & 6 & 1.689 & 2.041 & $85^{\star \star \star}$ & 6 & 0.027 & $0.02675^{\star \star \star}$ \\
\hline 12 & 0.000 & 0.001 & $55^{\star \star}$ & 12 & 0.019 & 0.057 & $61^{\star \star \star}$ & 12 & 0.003 & $0.00379^{\star \star \star}$ \\
\hline 3 & 0.000 & 0.000 & 52 & 3 & -0.015 & 0.043 & $56^{\star \star}$ & 3 & 0.002 & $0.00164^{\star \star \star}$ \\
\hline 8 & 0.010 & 0.008 & $59^{\star \star \star}$ & 8 & 1.025 & 1.303 & $77^{\star \star \star}$ & 8 & 0.018 & $0.01771^{\text {** }}$ \\
\hline 4 & 0.009 & 0.007 & $59^{\star \star \star *}$ & 4 & 0.467 & 0.697 & $69^{\star \star *}$ & 4 & 0.008 & $0.00762^{\star \star \star}$ \\
\hline 2 & 0.015 & 0.010 & $60^{\star \star \star}$ & 2 & 0.398 & 0.668 & $70^{\star \star \star}$ & 2 & 0.026 & $0.02284^{\star \star \star}$ \\
\hline 5 & 0.064 & 0.054 & $75^{\star \star \star}$ & 5 & 2.541 & 2.802 & $90^{\star \star \star}$ & 5 & 0.063 & $0.05988^{\star \star \star}$ \\
\hline 7 & 0.097 & $\begin{array}{l}0.088 \\
\text { CRPS }\end{array}$ & $79^{\star \star \star}$ & 7 & 4.116 & $\begin{array}{l}4.440 \\
\text { CRPS }\end{array}$ & $94^{\star \star \star}$ & 7 & 0.105 & $\begin{array}{l}0.09793^{\star \star *} \\
\text { CRPS }\end{array}$ \\
\hline 11 & 0.021 & 0.007 & $72^{\star \star \star}$ & 11 & 0.015 & 0.007 & $80^{\star \star \star}$ & 11 & -0.011 & -0.00247 \\
\hline 9 & 0.010 & 0.004 & $69^{\star \star \star \star}$ & 9 & 0.016 & 0.008 & $82^{\star \star \star}$ & 9 & -0.012 & 0.00050 \\
\hline 6 & -0.002 & -0.001 & $42^{\star \star \star}$ & 6 & -0.012 & -0.005 & $27^{\star \star \star}$ & 6 & 0.031 & $0.01476^{\star \star \star}$ \\
\hline 12 & 0.000 & 0.000 & 47 & 12 & -0.001 & 0.000 & $46^{*}$ & 12 & 0.001 & $0.00154^{*}$ \\
\hline 3 & -0.001 & -0.001 & $42^{\star \star \star}$ & 3 & -0.005 & -0.003 & $26^{\star \star \star}$ & 3 & 0.006 & $0.00257^{\star \star \star}$ \\
\hline 8 & -0.002 & -0.001 & $42^{\star \star \star}$ & 8 & -0.012 & -0.005 & $25^{\star \star \star}$ & 8 & 0.028 & $0.01373^{* \star \star}$ \\
\hline 4 & -0.002 & -0.002 & $41^{\star \star \star}$ & 4 & -0.012 & -0.006 & $23^{\star \star *}$ & 4 & 0.028 & $0.01271^{\star \star \star}$ \\
\hline 2 & 0.001 & 0.001 & $54^{\star}$ & 2 & -0.002 & 0.000 & 48 & 2 & 0.001 & $0.00458^{\star \star}$ \\
\hline 5 & 0.001 & 0.001 & $54^{\star}$ & 5 & -0.010 & -0.003 & $33^{\star \star \star}$ & 5 & 0.022 & $0.01371^{\star \star \star}$ \\
\hline 7 & 0.002 & $\begin{array}{l}0.002 \\
\text { LPS }\end{array}$ & $57^{\star \star \star}$ & 7 & -0.009 & $\begin{array}{l}-0.003 \\
\text { LPS }\end{array}$ & $38^{\star \star \star}$ & 7 & 0.027 & $\begin{array}{l}0.01674^{\star \star \star} \\
\text { LPS }\end{array}$ \\
\hline 11 & 0.022 & 0.057 & $56^{\star \star}$ & 11 & 0.114 & 0.120 & $70^{\star \star \star}$ & 11 & 0.443 & 0.46381 *** \\
\hline 9 & 0.044 & 0.053 & $55^{\star \star}$ & 9 & 0.127 & 0.127 & $71^{\star \star \star}$ & 9 & 0.279 & $0.33179^{\star \star \star}$ \\
\hline 6 & 1.076 & 1.542 & $76^{\star \star \star}$ & 6 & 1.689 & 2.041 & $85^{\star \star \star}$ & 6 & 3.885 & $2.73477^{\star \star \star}$ \\
\hline 12 & -0.091 & 0.030 & 52 & 12 & 0.019 & 0.057 & $61^{\star \star \star}$ & 12 & -0.418 & 0.01651 \\
\hline 3 & -0.114 & 0.049 & $56^{\star \star}$ & 3 & -0.015 & 0.043 & $56^{\star \star}$ & 3 & -0.543 & $-0.09245^{\star *}$ \\
\hline 8 & 0.414 & 0.817 & $71^{\star \star \star}$ & 8 & 1.025 & 1.303 & $77^{\star \star \star}$ & 8 & 2.955 & $1.87273^{\star \star \star}$ \\
\hline 4 & 0.374 & 0.768 & $71^{\star \star \star}$ & 4 & 0.467 & 0.697 & 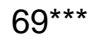 & 4 & 1.784 & $0.95467^{\star \star \star}$ \\
\hline 2 & 0.428 & 0.849 & $70^{\star \star \star}$ & 2 & 0.398 & 0.668 & $70^{\star \star \star \star}$ & 2 & 2.824 & $1.47576^{\star \star \star}$ \\
\hline 5 & 1.823 & 2.330 & $84^{\star \star \star}$ & 5 & 2.541 & 2.802 & $90^{\star \star \star \star}$ & 5 & 5.782 & $4.27487^{\star \star \star}$ \\
\hline 7 & 2.453 & 2.962 & $87^{\star \star \star \star}$ & 7 & 4.116 & 4.440 & $94^{\star \star \star}$ & 7 & 7.684 & $6.12492^{\star \star \star}$ \\
\hline
\end{tabular}

${ }^{\mathrm{a}}$ Refers to the improvement in the score attained by the UIP

${ }^{\mathrm{b}}$ Indicates percent of trials where "success" is a better predictive score by the UIP than by the alternative prior. $* * *, * * *$ are $99 \%, 95 \%, 90 \%$ significance levels based on binomial $\mathrm{p}$ values, $\mathrm{P}(\mathrm{X}>$ or $=\mathrm{z})$, for the given number of trials and successes; where success is defined as a better score for prior 1 (the alternative) as compared to the alternative prior (UIP) if the percentage is above (below) $50 \%$

1) Priors 9 and 10 are identical in the simulated dataset 
Figure 1

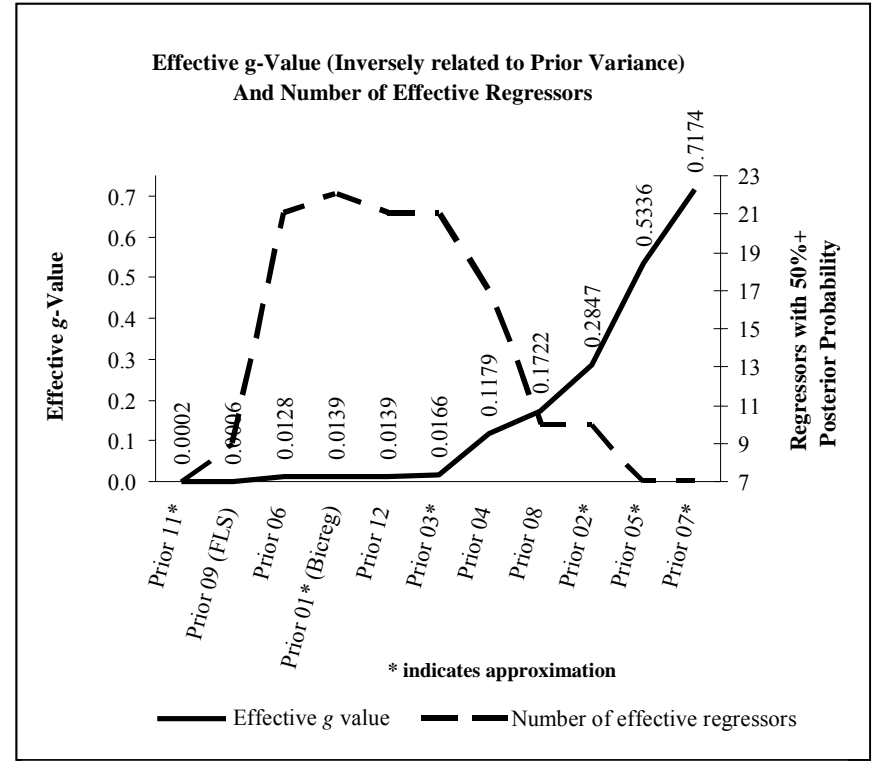

1) When priors depend on the exact model size, $p_{k}$, Figure 1 approximates the prior using the expected model size. Priors 11 and 1 are not exact $g$ priors, so the $g$ value is also an approximation

2) Priors 9 and 10 are identical in the growth context 
Figure 2

Correlation of Posterior Inclusion Probabilities Across Parameter Priors (Growth Dataset)
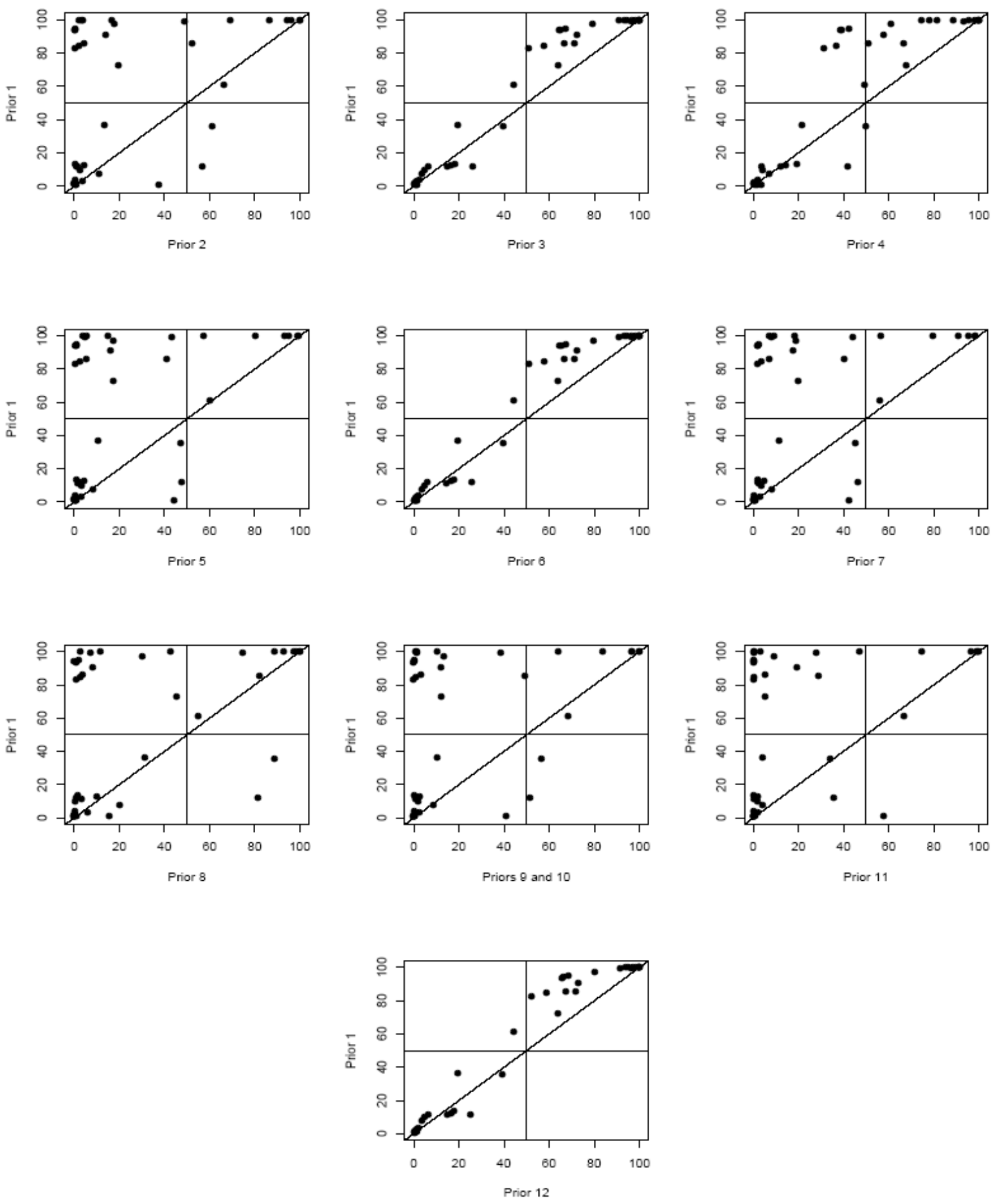

1) Priors 9 and 10 are identical in the growth context 
Figure 3

Regressors Included in Best Models

a) Prior 1 (uniform model prior)

Models selected by BMA

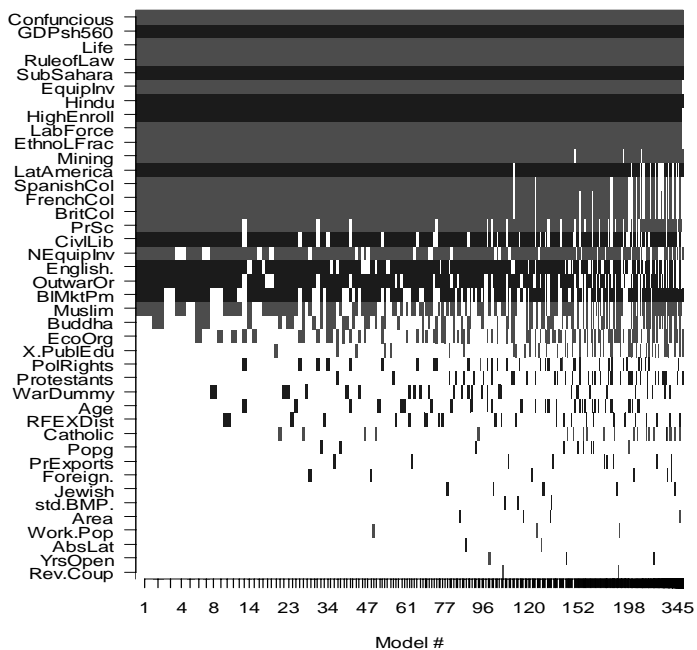

c) Prior 1 (prior model size $=7$ )

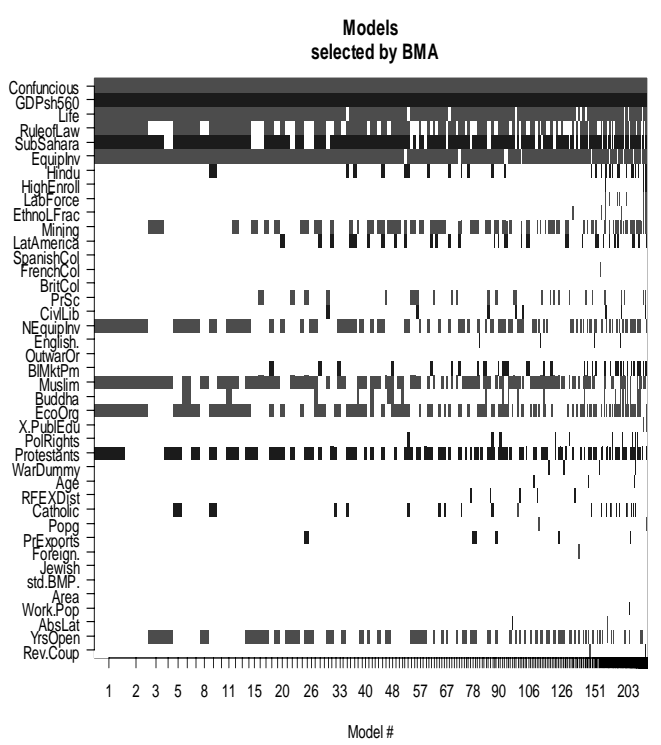

Notes: Posterior means are indicated as positive or negative (darker shading)

Horizontal distances indicate posterior model weights

Priors 9 and 10 are identical in the growth context. b) Prior 9 (uniform model prior)

Models selected by BMA

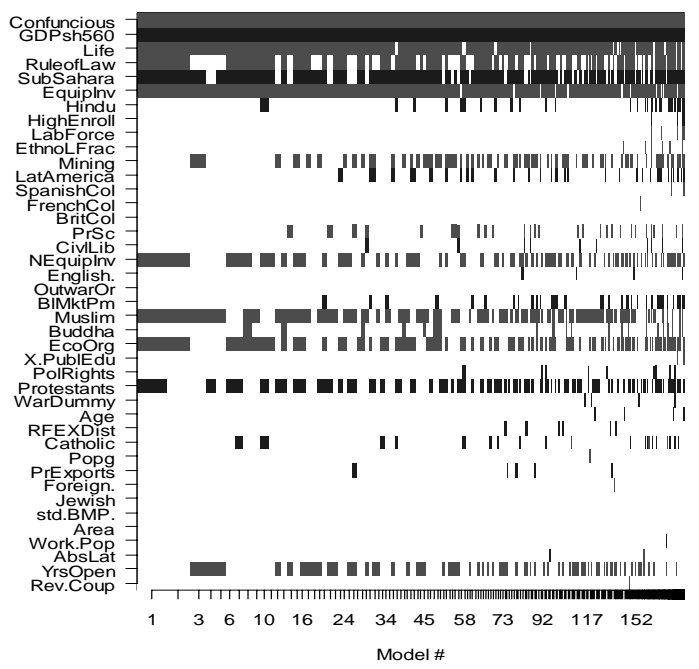




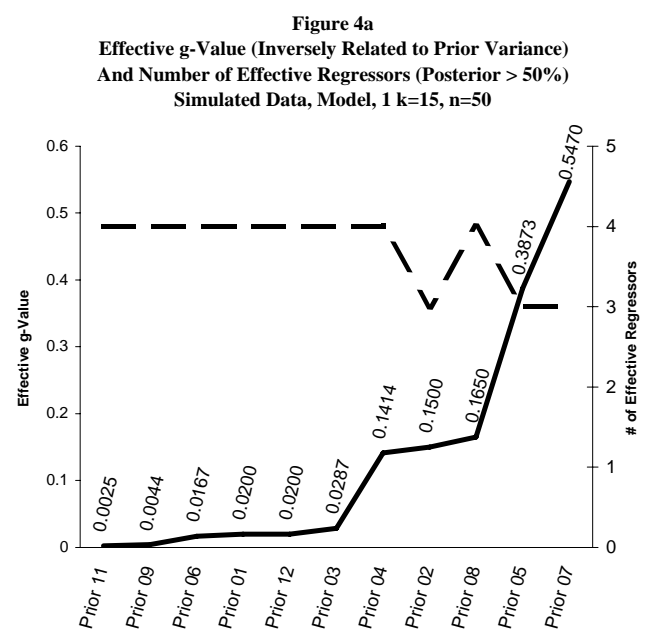

Figure $4 b$

Effective g-Value (Inversely Related to Prior Variance) And Number of Effective Regressors (Posterior $>50 \%$ ) Simulated Data, Model 1, k=15, $n=100$

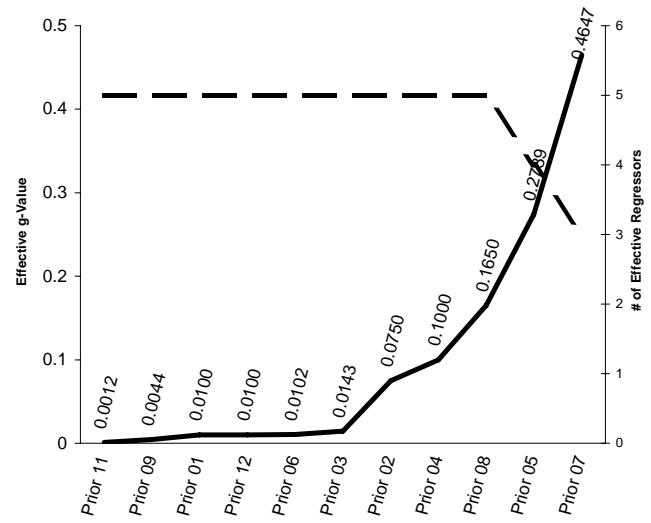

Figure 4c

Effective g-Value (Inversely Related to Prior Variance)

And Number of Effective Regressors (Posterior $>50 \%$ )

Simulated Data, Model 2, k=40, $n=100$

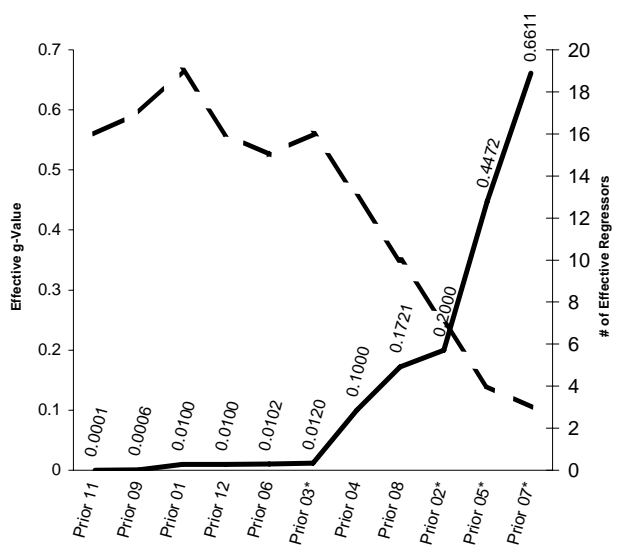

* indicates approximation

- Effective $g$ value $\quad$ - Pumber of effective regressors 9 -

1) Priors 9 and 10 are identical in the simulated datase

2) Priors 1 and 12 have the same g-value

3) Priors arranged by effective g-value (increasing left to righ

4) When priors depend on the exact model size, $p_{k}$, Figures 5a-c approximate the prior using the expected model size. Priors 11 and 1 are not exact $g$ priors, so the $g$ value is also an approximatio 